\title{
EXPECTED COALESCENCE TIME FOR A NONUNIFORM ALLOCATION PROCESS
}

\author{
JOHN K. MCSWEENEY *** AND \\ BORIS G. PITTEL, ${ }^{* * *}$ The Ohio State University
}

\begin{abstract}
We study a process where balls are repeatedly thrown into $n$ boxes independently according to some probability distribution $\boldsymbol{p}$. We start with $n$ balls, and at each step, all balls landing in the same box are fused into a single ball; the process terminates when there is only one ball left (coalescence). Let $c:=\sum_{j} p_{j}^{2}$, the collision probability of two fixed balls. We show that the expected coalescence time is asymptotically $2 c^{-1}$, under two constraints on $\boldsymbol{p}$ that exclude a thin set of distributions $\boldsymbol{p}$. One of the constraints is $c=o\left(\ln ^{-2} n\right)$. This $\ln ^{-2} n$ is shown to be a threshold value: for $c=\omega\left(\ln ^{-2} n\right)$, there exists $\boldsymbol{p}$ with $c(\boldsymbol{p})=c$ such that the expected coalescence time far exceeds $c^{-1}$. Connections to coalescent processes in population biology and theoretical computer science are discussed.
\end{abstract}

Keywords: Coalescence; most recent common ancestor; generalized Wright-Fisher model; random function; Markov chain; asymptotic behavior

2000 Mathematics Subject Classification: Primary 60C05

Secondary 60F10; 60J05; 68W40; 92D25

Remark. All limits in this paper are taken as $n \rightarrow \infty$, and we use Landau notation $o, \omega$, and $O$ in the usual sense. We say that an event $A$ holds with high probability (w.h.p.) if $\mathrm{P}\left(A^{\mathrm{c}}\right)=o(1)$, and we denote the set $\{1,2, \ldots, n\}$ by $[n]$.

\section{Introduction}

We consider the following balls-into-boxes process. Let $\boldsymbol{p}=\left(p_{1}, \ldots, p_{n}\right)$ be any probability vector. At time $t=0$, start with $b_{0}$ balls and throw them into $n$ boxes, where each ball has probability $p_{j}$ of landing in box $j$, independently of all other balls. Fuse all balls that land in the same box, and then repeat the allocation at times $t=1,2, \ldots$ according to the same rules with this possibly smaller new number of balls. The random time $T$ at which all balls are first fused into a single one is called the coalescence time; we will be mainly interested in its asymptotic expected value.

This problem has been studied in various guises by numerous authors. Initially, it was stated in terms of finding the most recent common ancestor in a random genealogical process. We will restrict ourselves to the balls-into-boxes formulation here, but it is important to note that any of the results we obtain here can be recast in the language of population biology; we will occasionally provide the reader with the appropriate analogy. The seminal work in this area

Received 15 August 2008; revision received 30 September 2008.

* Postal address: Department of Mathematics, The Ohio State University, $231 \mathrm{~W}$ 18th Avenue, Columbus, OH 43210, USA.

** Email address: mcsweeney@math.ohio-state.edu

*** Email address: bgp@math.ohio-state.edu 
was carried out by Kingman [7]-[9] who, for fixed $b_{0}$, proved convergence of the underlying process to the continuous-time coalescent process, thereby establishing convergence of the distribution of $T / \mathrm{E}[T]$. In [8] he also proved that, for $b_{0}=n$ and the uniform distribution $\boldsymbol{p}=(1 / n, \ldots, 1 / n)$,

$$
\mathrm{E}[T] \leq 2 n-2,
$$

which effectively implies that

$$
\mathrm{E}[T] \sim 2 n, \quad n \rightarrow \infty .
$$

More recently, Donnelly and Tavaré [3], Möhle [12], and Möhle and Sagitov [14] have studied the limiting behavior of more general classes of allocation (reproduction) models for $b_{0}$ fixed. In [13], Möhle considered the case $b_{0} \rightarrow \infty$ as well for several models, which, however, do not include the one at hand.

There is a good deal of literature on continuous-time coalescent processes that bears mentioning. For large times $t$, when the number of balls is small, we should expect relatively long time intervals during which no collisions happen, likely punctuated by binary (one-on-one) collisions. This behavior is characteristic of Kingman-type coalescent processes and admits a natural time scaling to a continuous-time process. More recently, the theory of $\Lambda$ - and $\Xi$-coalescents, developed by Pitman [15], Sagitov [19], and Schweinsberg [20], among others, allows for models involving multiple simultaneous collisions. Indeed, in our model, for small times $t$, we are likely to have multiple simultaneous collisions; however, these happen at fixed time intervals. There is therefore no natural time scaling that can be performed in order to interpret this behavior in the limit as a continuous-time process with random collision times.

The process can also be described in terms of compositions of random functions: choose random functions $\left\{f_{s}:[n] \rightarrow[n]\right\}_{s \in \mathbb{N}}$ independently, in such a way that, for all $i \in[n]$ and all $s \in \mathbb{N}, f_{s}(i)=j$ with probability $p_{j}$, independently for all $i$ and $s$. The coalescence time $T$ is then the smallest value of $t$ for which $f_{t} \circ \cdots \circ f_{2} \circ f_{1}$ is a constant function. It is this formulation that has been used in connection with computer science: this problem is potentially useful in bounding the running time of so-called 'coupling from the past' algorithms introduced in [17] and [18]; see Section 6 for a brief discussion of this. Motivated by this connection, and apparently unaware of Kingman's work for the uniform distribution, Dalal and Schmutz [2] established (1.1); Fill [4] and Goh et al. [6] derived the limiting distribution of $T / \mathrm{E}[T]$.

For $b_{0}=n$, Adler et al. [1, Theorem 4] were able to extend Kingman's result (1.1) to a nonuniform $\boldsymbol{p}$, showing that

$$
\mathrm{E}[T] \sim 2 c_{2}^{-1}, \quad c_{2}:=\sum_{j} p_{j}^{2}
$$

(note that $c_{2}=1 / n$ for the uniform $\boldsymbol{p}$ ), under the condition

$$
\frac{c_{3}}{c_{2}}<\frac{3}{n}, \quad c_{3}:=\sum_{j} p_{j}^{3} .
$$

Here $c_{2}$ and $c_{3}$ are the probabilities of a double collision and a triple collision, respectively. In essence, (1.3) means that $\boldsymbol{p}$ is sufficiently close to $(1 / n, \ldots, 1 / n)$.

We should expect the largest contribution to the time $T$ to happen during the late stages of the process, when the number of balls is relatively small. In this case, any reduction in the number of balls will most likely be due to the collision of a single pair of balls. This explains 
the appearance of $c_{2}$ in (1.2). (In fact, $c_{2}$ had been used as a scaling parameter for a wide class of models by Möhle in [12] in the context of population genetics.) The bound (1.3) on $c_{3}$ ensures that, if in one of those late stages the number of balls has dropped, then the actual decrease is exactly 1 with conditional probability sufficiently close to 1 .

The proof in [1] revealed that the expected time spent in the late stages was about $2 c_{2}^{-1}$ under conditions far less restrictive than (1.3). Equation (1.3) was used in [1] to show that the expected time spent in the early stages was $o\left(c_{2}^{-1}\right)$.

In this paper we prove that this property of the process and (1.2) continue to hold for a much wider class of distributions $\boldsymbol{p}$. Here is our main result.

Theorem 1.1. Let $b_{0}=n$. Suppose that, for some however small $\varepsilon>0$,

$$
c_{2}=o\left(\ln ^{-2} n\right) \quad \text { and } \quad c_{3} \leq c_{2}^{3 / 2} \ln ^{-(1 / 2+\varepsilon)} n .
$$

Then

(i)

$$
\mathrm{E}[T]=2 c_{2}^{-1}(1+o(1)) \text { for } n \rightarrow \infty,
$$

and

(ii)

$$
\frac{T}{\mathrm{E}[T]} \stackrel{\mathrm{D}}{\rightarrow} \sum_{k \geq 2} \frac{2}{k(k-1)} Y_{k},
$$

where $\stackrel{\mathrm{D}}{\rightarrow}$ ' denotes convergence in distribution, and the $Y_{k}$ are independent and exponentially distributed, i.e. $\mathrm{P}\left(Y_{k}>x\right)=\mathrm{e}^{-x}$.

Remarks. 1. It is always the case that $c_{2} \leq 1$ and $c_{3} \leq c_{2}^{3 / 2}$; so, if not for the logarithmic factors, the conditions in (1.4) would not have excluded any probability vectors $\boldsymbol{p}$. Furthermore, $c_{2}^{3 / 2} /\left(n^{-1} c_{2}\right) \geq n^{1 / 2}$, since $c_{2} \geq n^{-1}$ for all $\boldsymbol{p}$. This means that the restriction $c_{3} / c_{2}<3 / n$ in [1] is much more stringent than $c_{3} \leq c_{2}^{3 / 2} \ln ^{-(1 / 2+\varepsilon)} n$ in our Theorem 1.1.

2. The lower bound in (1.5), $\mathrm{E}[T] \geq 2 c_{2}^{-1}(1-o(1))$, holds for any $\boldsymbol{p}$ as long as $c_{2} \rightarrow 0$. This can be deduced from [1, Theorem 2] via an elementary coupling argument; we will provide a brief proof in Section 4. Therefore, our main task is to prove a matching upper bound.

3. For the case of the uniform $p=(1 / n, \ldots, 1 / n)$, (1.6) follows from Kingman's work. Independently, it was proved later-in a setup close to that of our paper-by Fill [4]; still later Goh et al. [6] gave a detailed description of the cumulative distribution function of the limiting distribution.

4. Part (ii) of Theorem 1.1 follows from part (i), via an argument very similar to Fill's proof for the uniform case; $\mathrm{cf}$. Theorem 6.1 of [13].

Interestingly, $\ln ^{-2} n$ appearing as the upper bound for $c_{2}$ in Theorem 1.1 is a genuine threshold for the property 'E[T] is of order $c_{2}^{-1}$ exactly'.

Theorem 1.2. Let $b_{0}=n$. For $c_{2}=\omega\left(\ln ^{-2} n\right)$, there exists a probability vector $\boldsymbol{p}$ with $\sum_{j} p_{j}^{2}=c_{2}$ such that, w.h.p., $T=\omega\left(c_{2}^{-1}\right)$, and, thus, $\mathrm{E}[T]=\omega\left(c_{2}^{-1}\right)$.

Adler et al. [1, Theorem 5] proved that $\mathrm{E}[T] \rightarrow \infty$ for a probability vector satisfying conditions ensuring that $\lim c_{2}>0$. 
The rest of the paper is organized as follows. In Section 2 we bound the expected time spent during an early phase. We achieve this by showing that, w.h.p., for certain 'small' values of $t$, the stochastic process is well approximated by a deterministic process, amenable to sharp estimates. To this end, we will build on the method used in [16] for asymptotic analysis of a rumor-spreading process introduced and studied in [5]. In Section 3 we bound the expected time spent in a 'middle' phase and a 'late' phase, showing that the late phase contributes, overwhelmingly, to the total number of steps. In Section 4 we prove the lower bound for Theorem 1.1. In Section 5 we prove Theorem 1.2. In Appendix A we prove some auxiliary inequalities needed for the proof of Theorem 1.1.

\section{The expected duration of an early phase}

Let the distribution $\boldsymbol{p}$ be given. Assume that $b_{0}=n$. For $t \in \mathbb{N}$, we denote by $B(t)$ the random number of balls at time $t$, so that $B(0)=n$. In the language of the genealogical process, $B(t)$ is the number of individuals at generation $-t$ which have a descendant alive in the current generation 0 . We denote by $\tau(k)$ the random first time $t$ when $B(t)$ falls below $k$, i.e. $\tau(k)=\min \{t \geq 0: B(t) \leq k\}$. The coalescence time $T$ is therefore $\tau(1)$. Obviously, $\{B(t)\}_{t \geq 0}$ is a Markov chain on the state space $\{1,2, \ldots, n\}$, so we will refer to $B(t)$ as the state at time $t$.

Note that, by the definition of the stochastic sequence $\{B(t)\}_{t \geq 0}$,

$$
\mathrm{E}[B(t+1) \mid B(t)=k]=\sum_{j=1}^{n}\left(1-\left(1-p_{j}\right)^{k}\right), \quad B(0)=n,
$$

because the probability of box $j$ receiving at least one ball out of $k$ allocated balls is $1-\left(1-p_{j}\right)^{k}$. It would seem natural to try to prove that the conditional distribution of $B(t+1)$ is concentrated around $\mathrm{E}[B(t+1) \mid B(t)=k]$, as long as $k$ is large enough. Curiously, we will be able to show instead that, for smallish $t$, w.h.p., $B(t+1)$ is relatively close to $\Phi_{p}(B(t))$, where

$$
\Phi_{p}(k):=\sum_{j=1}^{n}\left(1-\exp \left(-p_{j} k\right)\right) .
$$

Note that $\Phi_{p}(B(t))$ is close to $\mathrm{E}[B(t+1) \mid B(t)]$ when most of the $p_{j} B(t)$ are small, which may not be the case when $B(t)$ is relatively close to $n$.

Here is an outline of our argument. We introduce $k_{*}=o\left(c_{2}^{-1}\right)$, and a recurrence inequality which the random sequence $B(t)$ is believed to satisfy w.h.p. as long as $B(t)$ is above $k_{*}$. Assuming that the inequality does hold, we derive efficient bounds for $\tau\left(k_{*}\right)$. Lastly, we show that, indeed w.h.p., $\{B(t)\}_{t \leq \tau\left(k_{*}\right)}$ satisfies the recurrence inequality.

\subsection{Variational problems}

In order to determine the likely decline of $\{B(t)\}$, we need to bound $\Phi_{p}(k)$ from above for a certain range of $k$. This task seems quite hard, since $\Phi_{p}(k)$ depends on all $n$ components of $\boldsymbol{p}$ in a rather complicated way. Remarkably, the worst case bounds will do the job quite efficiently, because the worst distribution $\boldsymbol{p}$ turns out to be much simpler than a feasible generic $\boldsymbol{p}$.

Let us define $D_{n}$ to be the set of probability $n$-vectors. For any $\boldsymbol{q} \in D_{n}$, define

$$
F_{\boldsymbol{q}}(k):=\sum_{j=1}^{n} \exp \left(-k q_{j}\right)
$$


so that $\Phi_{\boldsymbol{q}}(k)=n-F_{\boldsymbol{q}}(k)$, and set

$$
D\left(c_{2}\right):=\left\{\boldsymbol{q} \in D_{n} \mid \sum_{j} q_{j}^{2}=c_{2}\right\}
$$

and

$$
D\left(c_{2}, c_{3}\right):=\left\{\boldsymbol{q} \in D_{n} \mid \sum_{j} q_{j}^{2}=c_{2}, \sum_{j} q_{j}^{3}=c_{3}\right\} .
$$

That is, $D\left(c_{2}\right)$ and $D\left(c_{2}, c_{3}\right)$ are the sets of probability vectors that share the same sum of squares and, respectively, squares and cubes as $\boldsymbol{p}$. When dealing with $D\left(c_{2}\right)$ and $D\left(c_{2}, c_{3}\right)$, we will assume that $c_{2}>1 / n$; otherwise these sets are reduced to a point $(1 / n, \ldots, 1 / n)$. The functional $F_{q}(k)$ is continuous and the sets $D_{n}, D\left(c_{2}\right)$, and $D\left(c_{2}, c_{3}\right)$ are compact, so the infima of $F$ (as a function of $\boldsymbol{q}$ for fixed $k$ ) over these sets are attained.

Proposition 2.1. For any $k \in[n]$,

$$
\min _{\boldsymbol{q} \in D_{n}} F_{\boldsymbol{q}}(k)=F_{\boldsymbol{u}}(k),
$$

where $\boldsymbol{u}$ is the uniform vector $\boldsymbol{u}=(1 / n, \ldots, 1 / n)$.

Proposition 2.2. For any $k \in[n]$,

$$
\min _{\boldsymbol{q} \in D\left(c_{2}\right)} F_{\boldsymbol{q}}(k)=F_{\boldsymbol{\theta}\left(c_{2}\right)}(k),
$$

where $\boldsymbol{\theta}\left(c_{2}\right)=\left(\theta_{1}, \theta_{2}, \ldots, \theta_{n}\right)$ has the property that $\theta_{1}>\theta_{2}=\cdots=\theta_{n}$ (when listed in nonincreasing order). That is, $\boldsymbol{\theta}$ has only two distinct entries, and the larger one has support size equal to 1 . We will refer to such vectors as being of 'topheavy' type. Using the equations $\theta_{1}+(n-1) \theta_{2}=1$ and $\theta_{1}^{2}+(n-1) \theta_{2}^{2}=c_{2}$, we can explicitly express the two entries of $\boldsymbol{\theta}$ as

$$
\theta_{1}=\frac{1+\sqrt{(n-1)\left(c_{2} n-1\right)}}{n}, \quad \theta_{2}=\frac{1-\theta_{1}}{n-1} .
$$

Proposition 2.3. For any $k \in[n]$,

$$
\min _{\boldsymbol{q} \in D\left(c_{2}, c_{3}\right)} F_{\boldsymbol{q}}(k)=F_{\boldsymbol{r}\left(c_{2}, c_{3}\right)}(k)
$$

where $\boldsymbol{r}\left(c_{2}, c_{3}\right):=\left(r_{1}, r_{2}, \ldots, r_{n}\right)$ (when ordered in nonincreasing order) has the following property: for some $v \in[n]$,

$$
r_{1}=\cdots=r_{v} \geq r_{v+1}>r_{v+2}=\cdots=r_{n}
$$

That is, $\boldsymbol{r}\left(c_{2}, c_{3}\right)$ has at most three distinct entries, and the middle one (if any) has support size equal to 1 .

Clearly, $D_{n} \supset D\left(c_{2}\right) \supset D\left(c_{2}, c_{3}\right)$, and so $F_{\boldsymbol{p}}(k) \geq F_{\boldsymbol{r}\left(c_{2}, c_{3}\right)}(k) \geq F_{\boldsymbol{\theta}\left(c_{2}\right)}(k) \geq F_{\boldsymbol{u}}(k)$. These propositions therefore provide sharper and sharper estimates, so at various junctures we will use whichever one is easiest to work with, while still being sharp enough. 
Proof of Proposition 2.1. Using the fact that $\varphi(x)=\mathrm{e}^{-x}$ is concave up,

$$
F_{\boldsymbol{p}}(k)=\sum_{j} \exp \left(-p_{j} k\right)=n \sum_{j} \frac{1}{n} \exp \left(-p_{j} k\right) \geq n \exp \left(-\sum_{j} \frac{1}{n} p_{j} k\right)=n \mathrm{e}^{-k / n}=F_{\boldsymbol{u}}(k) .
$$

Proofs of Propositions 2.2 and 2.3. To prove Proposition 2.2, there are two steps.

1. Show that a minimizer $\boldsymbol{\theta}$ of $F_{\boldsymbol{q}}(k)$ on $D\left(c_{2}\right)$ cannot have a configuration $\theta_{j_{1}}>\theta_{j_{2}}>\theta_{j_{3}}$; that is, that it cannot have three distinct entries.

2. Show that a minimizer $\boldsymbol{\theta}$ cannot have a $\theta_{j_{1}}=\theta_{j_{2}}>\theta_{j_{3}}$ configuration $\left(j_{i} \in[n]\right)$, which will imply that the larger entry is unique.

We will only prove Proposition 2.3, as it is more difficult; the interested reader would not find it difficult to adapt the argument to prove Proposition 2.2.

We may recast Proposition 2.3 as follows: letting $z_{j}:=q_{j} k$ and $z=\left(z_{1}, \ldots, z_{n}\right)$, we want to minimize $G(z):=\sum_{j} \exp \left(-z_{j}\right)$ under the constraints

$$
\sum_{j} z_{j}=k, \quad \sum_{j} z_{j}^{2}=k^{2} c_{2}, \quad \text { and } \quad \sum_{j} z_{j}^{3}=k^{3} c_{3} .
$$

Our task is to show that the minimizer $\boldsymbol{x}=\left(x_{1}, \ldots, x_{n}\right)$ of $G(z)$, with components listed in nonincreasing order, must have the form $x_{1}=\cdots=x_{v} \geq x_{v+1}>x_{v+2}=\cdots=x_{n}$ for some $v \in[n]$. To this end, we show first that a minimizer of $G(z)$ has at most four distinct components (see case I, below) and second that an entry value which is strictly intermediate is encountered exactly once (see case II, below). Our proof does not rely on the method of Lagrange multipliers, because its applicability for the equality constraints needs a prior justification and because, in principle, it may deliver only a 'first-order' necessary condition, definitely too crude to handle case II.

Case I. We first show that a minimizer of $G(z)$ cannot have four distinct entries. Suppose for the sake of contradiction that we have a minimizing vector $\boldsymbol{x}$ for which there exist $j_{1}, j_{2}, j_{3}$, and $j_{4}$ (relabel as 1,2,3, and 4) such that $x_{1}>x_{2}>x_{3}>x_{4} \geq 0$. Let $y_{j}=x_{j}+\varepsilon_{j}$ for $j=1, \ldots, 4$, and $y_{j}=x_{j}$ for $j=5, \ldots, n$; we will show that, for a suitable choice of $\left(\varepsilon_{1}, \ldots, \varepsilon_{4}\right), \boldsymbol{y}=\left(y_{1}, \ldots, y_{n}\right)$ satisfies the conditions in (2.5), and $G(\boldsymbol{y})<G(\boldsymbol{x})$, and, thus, such an $\boldsymbol{x}$ cannot be a minimizer on the set $D\left(c_{2}, c_{3}\right)$.

First note that we require $\varepsilon_{4} \geq 0$ because of the possibility that $x_{4}=0$, but $\varepsilon_{1}, \varepsilon_{2}$, and $\varepsilon_{3}$ can be of either sign. For $y$ to satisfy the conditions in (2.5), we require that

$$
\begin{gathered}
\sum_{j=1}^{4} \varepsilon_{j}=0, \\
2 \sum_{j=1}^{4} x_{j} \varepsilon_{j}+\sum_{j=1}^{4} \varepsilon_{j}^{2}=0, \\
3 \sum_{j=1}^{4} x_{j}^{2} \varepsilon_{j}+3 \sum_{j=1}^{4} x_{j} \varepsilon_{j}^{2}+\sum_{j=1}^{4} \varepsilon_{j}^{3}=0 .
\end{gathered}
$$

Now we want $G(\boldsymbol{x})-G(\boldsymbol{y})>0$; by linearizing the $\mathrm{e}^{-\varepsilon_{j}}$ factors, it will be sufficient (by taking the $\varepsilon_{j}$ as small as we wish) to show that

$$
\mathrm{e}^{-x_{1}} \varepsilon_{1}+\mathrm{e}^{-x_{2}} \varepsilon_{2}+\mathrm{e}^{-x_{3}} \varepsilon_{3}+\mathrm{e}^{-x_{4}} \varepsilon_{4}>0 .
$$


We now obtain expressions for the $\varepsilon_{j}$. For given $x_{j}$, the system (2.6)-(2.8) is a system of three nonlinear equations in four unknowns, $\varepsilon_{1}, \varepsilon_{2}, \varepsilon_{3}$, and $\varepsilon_{4}$; treating $\varepsilon_{4}$ as a parameter, we hope to be able to solve it uniquely for $\varepsilon_{1}, \varepsilon_{2}$, and $\varepsilon_{3}$ near $(0,0,0)^{\top}$. Let $\boldsymbol{\varepsilon}:=\left(\varepsilon_{1}, \varepsilon_{2}, \varepsilon_{3}\right)^{\top}$, and write (2.6)-(2.8) as the vector equation:

$$
\boldsymbol{f}(\boldsymbol{\varepsilon})=\boldsymbol{b}\left(\varepsilon_{4}\right), \quad \boldsymbol{b}\left(\varepsilon_{4}\right):=\left(-\varepsilon_{4},-2 x_{4} \varepsilon_{4}-\varepsilon_{4}^{2},-3 x_{4}^{2} \varepsilon_{4}-3 x_{4} \varepsilon_{4}^{2}-\varepsilon_{4}^{3}\right)^{\top} .
$$

The derivative (Jacobian) matrix of $\boldsymbol{f}$ at $\mathbf{0}=(0,0,0)$ is

$$
\boldsymbol{L}:=\left(\begin{array}{ccc}
1 & 1 & 1 \\
2 x_{1} & 2 x_{2} & 2 x_{3} \\
3 x_{1}^{2} & 3 x_{2}^{2} & 3 x_{3}^{2}
\end{array}\right)
$$

Its determinant is equal to $6 \Delta\left(x_{1}, x_{2}, x_{3}\right)$, where $\Delta\left(x_{1}, x_{2}, x_{3}\right)$ is the Vandermonde determinant for $x_{1}, x_{2}$, and $x_{3}$ :

$$
\Delta\left(x_{1}, x_{2}, x_{3}\right)=\left(x_{2}-x_{1}\right)\left(x_{3}-x_{1}\right)\left(x_{3}-x_{2}\right),
$$

which is nonzero (negative), as the $x_{i}$ are distinct (decreasing). Therefore, by the inverse vector function theorem, for sufficiently small $\left|\varepsilon_{4}\right|$, there exists a differentiable solution $\boldsymbol{\varepsilon}=\boldsymbol{\varepsilon}\left(\varepsilon_{4}\right)$, $\boldsymbol{\varepsilon}(0)=\mathbf{0}$, such that

$$
\boldsymbol{\varepsilon}=\boldsymbol{\gamma} \varepsilon_{4}+\boldsymbol{O}\left(\varepsilon_{4}^{2}\right), \quad \boldsymbol{\gamma}:=\boldsymbol{L}^{-1} \boldsymbol{b}^{\prime}(0)=\boldsymbol{L}^{-1}\left(-1,-2 x_{4},-3 x_{4}^{2}\right)^{\top} .
$$

Here $\boldsymbol{O}\left(\varepsilon_{4}^{2}\right)=\left(O\left(\varepsilon_{4}^{2}\right), O\left(\varepsilon_{4}^{2}\right), O\left(\varepsilon_{4}^{2}\right)\right)$. Explicitly, by Cramer's rule,

$$
\gamma_{1}=-\frac{\Delta\left(x_{4}, x_{2}, x_{3}\right)}{\Delta\left(x_{1}, x_{2}, x_{3}\right)}, \quad \gamma_{2}=-\frac{\Delta\left(x_{1}, x_{4}, x_{3}\right)}{\Delta\left(x_{1}, x_{2}, x_{3}\right)}, \quad \gamma_{3}=-\frac{\Delta\left(x_{1}, x_{2}, x_{4}\right)}{\Delta\left(x_{1}, x_{2}, x_{3}\right)} .
$$

With these formulae, (2.9) is equivalent to showing (by letting $\varepsilon_{4}>0$ be as small as needed) that

$$
\mathrm{e}^{-x_{4}} \Delta\left(x_{1}, x_{2}, x_{3}\right)<\mathrm{e}^{-x_{1}} \Delta\left(x_{4}, x_{2}, x_{3}\right)+\mathrm{e}^{-x_{2}} \Delta\left(x_{1}, x_{4}, x_{3}\right)+\mathrm{e}^{-x_{3}} \Delta\left(x_{1}, x_{2}, x_{4}\right),
$$

which in turn is equivalent to showing that

$$
D(x):=\left|\begin{array}{cccc}
\mathrm{e}^{-x_{1}} & \mathrm{e}^{-x_{2}} & \mathrm{e}^{-x_{3}} & \mathrm{e}^{-x_{4}} \\
1 & 1 & 1 & 1 \\
x_{1} & x_{2} & x_{3} & x_{4} \\
x_{1}^{2} & x_{2}^{2} & x_{3}^{2} & x_{4}^{2}
\end{array}\right|>0 .
$$

By using the operations typical for computation of the Vandermonde-type determinants, we obtain

$$
\begin{aligned}
D(\mathbf{x}) & =-\left|\begin{array}{ccc}
\mathrm{e}^{-x_{2}}-\mathrm{e}^{-x_{1}} & \mathrm{e}^{-x_{3}}-\mathrm{e}^{-x_{1}} & \mathrm{e}^{-x_{4}}-\mathrm{e}^{-x_{1}} \\
x_{2}-x_{1} & x_{3}-x_{1} & x_{4}-x_{1} \\
x_{2}^{2}-x_{1}^{2} & x_{3}^{2}-x_{1}^{2} & x_{4}^{2}-x_{1}^{2}
\end{array}\right| \\
& =\mathrm{e}^{-x_{1}} \prod_{i=2}^{4}\left(x_{i}-x_{1}\right)\left|\begin{array}{cc}
\lambda\left(x_{1}-x_{2}\right)-\lambda\left(x_{1}-x_{3}\right) & \lambda\left(x_{1}-x_{2}\right)-\lambda\left(x_{1}-x_{4}\right) \\
x_{3}-x_{2} & x_{4}-x_{2}
\end{array}\right|,
\end{aligned}
$$


where $\lambda(x):=\left(\mathrm{e}^{x}-1\right) / x$. Next, we factor $x_{3}-x_{2}$ and $x_{4}-x_{2}$ from the first column and from the second column. So, introducing

$$
C(\boldsymbol{x}):=\mathrm{e}^{-x_{1}} \prod_{i=2}^{4}\left(x_{i}-x_{1}\right) \prod_{i=3}^{4}\left(x_{i}-x_{2}\right)<0,
$$

we then obtain

$$
\begin{aligned}
D(\boldsymbol{x}) & =C(\boldsymbol{x})\left[\begin{array}{cc}
\frac{\lambda\left(x_{1}-x_{2}\right)-\lambda\left(x_{1}-x_{3}\right)}{x_{3}-x_{2}} & \frac{\lambda\left(x_{1}-x_{2}\right)-\lambda\left(x_{1}-x_{4}\right)}{x_{4}-x_{2}} \\
1 & 1
\end{array} \mid\right. \\
& =C(\boldsymbol{x})\left[\frac{\lambda\left(x_{1}-x_{2}\right)-\lambda\left(x_{1}-x_{3}\right)}{\left(x_{1}-x_{2}\right)-\left(x_{1}-x_{3}\right)}-\frac{\lambda\left(x_{1}-x_{2}\right)-\lambda\left(x_{1}-x_{4}\right)}{\left(x_{1}-x_{2}\right)-\left(x_{1}-x_{4}\right)}\right] .
\end{aligned}
$$

Now $\lambda(x)$ is concave up for $x>0$. Therefore, since

$$
0<x_{1}-x_{2}<x_{1}-x_{3}<x_{1}-x_{4},
$$

the quantity in square brackets in (2.10) is strictly negative, by considering its terms to be slopes of secant lines to the graph of $\lambda(x)$. Using this and $C(\boldsymbol{x})<0$, we obtain the desired conclusion, i.e. $D(\boldsymbol{x})>0$.

Case II. Now we show that a vector $\boldsymbol{x}$ with a configuration $x_{1}>x_{2}=x_{3}>x_{4} \geq 0$ cannot be a minimizer of $G$ either. Define $x:=x_{2}=x_{3}$. Now that $f^{\prime}(\mathbf{0})$ is singular, determination of small feasible $\varepsilon_{1}, \ldots, \varepsilon_{4}$ such that $G(\boldsymbol{y})<G(\boldsymbol{x})$ is more of a challenge. The fact that the linear terms in (2.6)-(2.8) now depend on only $\varepsilon_{1}, \varepsilon_{2}+\varepsilon_{3}$, and $\varepsilon_{4}$ hints that $\left|\varepsilon_{1}\right|,\left|\varepsilon_{2}+\varepsilon_{3}\right|$, and $\left|\varepsilon_{4}\right|$ should be equally small, and that $\left|\varepsilon_{2}\right|$ and $\left|\varepsilon_{3}\right|$, while small, should be much larger.

Believing in this scenario, we set

$$
\varepsilon_{1}=\delta_{1} \varepsilon^{2}, \quad \varepsilon_{2}=\varepsilon+\delta_{2} \varepsilon^{2}, \quad \varepsilon_{3}=-\varepsilon, \quad \varepsilon_{4}=\delta_{4} \varepsilon^{2},
$$

and seek the feasible $\delta_{i}(\varepsilon)$ for small $\varepsilon$. To begin with, we again require that $\delta_{4} \geq 0$. Letting $\boldsymbol{\delta}=\left(\delta_{1}, \delta_{2}, \delta_{4}\right)$, conditions (2.6)-(2.8) become

$$
\begin{aligned}
\delta_{1}+\delta_{2}+\delta_{4} & =0, \\
x_{1} \delta_{1}+x \delta_{2}+x_{4} \delta_{4} & =-1+\varepsilon b_{2}(\varepsilon, \delta), \\
x_{1}^{2} \delta_{1}+x^{2} \delta_{2}+x_{4}^{2} \delta_{4} & =-2 x+\varepsilon b_{3}(\varepsilon, \delta),
\end{aligned}
$$

where the $b_{i}(\varepsilon, \delta)$ are polynomials. Note that $\Delta:=\Delta\left(x_{1}, x, x_{4}\right)$, the determinant of the matrix in (2.11)-(2.13), is nonzero. So, for small enough $|\varepsilon|$, there exists a differentiable solution $\delta(\varepsilon)$ such that $\delta(0)$ is the solution of (2.11)-(2.13) with $0,-1$, and $-2 x$ respectively on the right-hand side. By Cramer's rule,

$$
\begin{gathered}
\delta_{1}(0)=\frac{1}{\Delta}\left|\begin{array}{ccc}
0 & 1 & 1 \\
-1 & x & x_{4} \\
-2 x & x^{2} & x_{4}^{2}
\end{array}\right|, \quad \delta_{2}(0)=\frac{1}{\Delta}\left|\begin{array}{ccc}
1 & 0 & 1 \\
x_{1} & -1 & x_{4} \\
x_{1}^{2} & -2 x & x_{4}^{2}
\end{array}\right|, \\
\delta_{4}(0)=\frac{1}{\Delta}\left|\begin{array}{ccc}
1 & 1 & 0 \\
x_{1} & x & -1 \\
x_{1}^{2} & x^{2} & -2 x
\end{array}\right|,
\end{gathered}
$$


which gives

$$
\delta_{1}(0)=\frac{\left(x-x_{4}\right)^{2}}{\Delta}, \quad \delta_{2}(0)=\frac{\left(x_{1}-x_{4}\right)\left(x_{1}+x_{4}-2 x\right)}{\Delta}, \quad \delta_{4}(0)=-\frac{\left(x-x_{1}\right)^{2}}{\Delta} .
$$

Reassuringly, $\delta_{4}(0)$ is positive (because $\Delta$ is negative). Again, we want $G(\boldsymbol{x})-G(\boldsymbol{x}+\boldsymbol{\varepsilon})>0$; we have

$$
\begin{aligned}
G(\boldsymbol{x})-G(\boldsymbol{x}+\varepsilon)= & \sum_{j=1}^{4}\left(1-\mathrm{e}^{-\varepsilon_{j}}\right) \mathrm{e}^{-x_{j}} \\
= & \mathrm{e}^{-x_{1}}\left(1-\mathrm{e}^{-\varepsilon_{1}}\right)+\mathrm{e}^{-x}\left(1-\mathrm{e}^{-\varepsilon_{2}}+1-\mathrm{e}^{-\varepsilon_{3}}\right)+\mathrm{e}^{-x_{4}}\left(1-\mathrm{e}^{-\varepsilon_{4}}\right) \\
= & \mathrm{e}^{-x_{1}}\left(\delta_{1} \varepsilon^{2}+O\left(\varepsilon^{4}\right)\right) \\
& +\mathrm{e}^{-x}\left(\varepsilon+\delta_{2} \varepsilon^{2}-\frac{1}{2}\left(\varepsilon+\delta_{2} \varepsilon^{2}\right)^{2}+O\left(\varepsilon^{3}\right)-\varepsilon-\frac{1}{2} \varepsilon^{2}+O\left(\varepsilon^{3}\right)\right) \\
& +\mathrm{e}^{-x_{4}}\left(\delta_{4} \varepsilon^{2}+O\left(\varepsilon^{4}\right)\right) \\
= & \varepsilon^{2}\left(\delta_{1} \mathrm{e}^{-x_{1}}+\left(\delta_{2}-1\right) \mathrm{e}^{-x}+\delta_{4} \mathrm{e}^{-x_{4}}+O(\varepsilon)\right) .
\end{aligned}
$$

Thus, by taking $\varepsilon$ sufficiently small, (2.14) will be greater than 0 if

$$
\delta_{1}(0) \mathrm{e}^{-x_{1}}+\left(\delta_{2}(0)-1\right) \mathrm{e}^{-x}+\mathrm{e}^{-x_{4}} \delta_{4}(0)>0 .
$$

In light of the formulae for the $\delta_{i}(0)$ and the fact that $\Delta<0$, this is equivalent to

$$
T(\boldsymbol{x}):=-\left(x-x_{4}\right)^{2} \mathrm{e}^{-x_{1}}+\left(\Delta-\left(x_{1}-x_{4}\right)\left(x_{1}+x_{4}-2 x\right)\right) \mathrm{e}^{-x}+\left(x-x_{1}\right)^{2} \mathrm{e}^{-x_{4}}>0 .
$$

Now multiplying $T(\boldsymbol{x})$ by $\mathrm{e}^{x}$ and using the inequalities

$$
\exp \left(-\left(x_{1}-x\right)\right)<1-\left(x_{1}-x\right)+\frac{\left(x_{1}-x\right)^{2}}{2}, \quad \exp \left(x-x_{4}\right)>1+\left(x-x_{4}\right)+\frac{\left(x-x_{4}\right)^{2}}{2}
$$

we obtain

$$
\begin{aligned}
\mathrm{e}^{x} T(\boldsymbol{x}) & =\left(x-x_{4}\right)^{2}\left(1-\exp \left(x-x_{1}\right)\right)+\left(x-x_{1}\right)^{2}\left(\exp \left(x-x_{4}\right)-1\right)+\Delta \\
& >\left(x-x_{4}\right)^{2}\left(\left(x_{1}-x\right)-\frac{\left(x_{1}-x\right)^{2}}{2}\right)+\left(x-x_{1}\right)^{2}\left(\left(x-x_{4}\right)+\frac{\left(x-x_{4}\right)^{2}}{2}\right)+\Delta \\
& =\left(x-x_{4}\right)^{2}\left(x_{1}-x\right)+\left(x-x_{1}\right)^{2}\left(x-x_{4}\right)+\left(x-x_{1}\right)\left(x_{4}-x_{1}\right)\left(x_{4}-x\right) \\
& =0 .
\end{aligned}
$$

Therefore, (2.15) holds, and, thus, as before, $\boldsymbol{x}$ cannot be a minimizer. This concludes case II.

This only leaves the possibility that the minimizer $x$ of $G$ is of the form

$$
x_{1}=x_{2}=\cdots=x_{v} \geq x_{v+1} \geq x_{v+2}=\cdots=x_{n},
$$

and, thus, that the minimizer $\boldsymbol{r}$ of $F_{\boldsymbol{q}}(k)$ over $D\left(c_{2}, c_{3}\right)$ is of the form (2.4) for any $k$. This concludes the proof of Proposition 2.3. 


\subsection{Identifying and iterating a likely recurrence inequality}

Let

$$
k_{*}:=c_{2}^{-1} \ln ^{-\varepsilon} n,
$$

with $\varepsilon$ coming from (1.4). (Here $k_{*}$ is meant to be an integer, as is another parameter $k_{1}$ defined later, but for simplicity we omit the 'integer part' notation.) This $k_{*}$ will serve as a threshold separating the 'early' states $\left(B(t)>k_{*}\right)$ from the 'late' states $\left(B(t) \leq k_{*}\right)$. So, in light of the informal discussion in the introduction, ' $k_{*}=o\left(c_{2}^{-1}\right)$ ' should be more or less expected; the need for an additional factor, $\ln ^{-\varepsilon} n$, will become clear later in Section 3 .

Our immediate task is to identify a function $\Psi_{p}(k)$ such that, intuitively at least, the random sequence $\{B(t)\}$, w.h.p., satisfies a recurrence inequality

$$
B(t+1) \leq \Psi_{p}(B(t)) \quad \text { if } B(t) \geq k_{*} .
$$

Then, for $k \geq k_{*}, \Psi_{p}(k)$ needs to be large enough so that, conditionally on $\{B(t)=k\}$, the event $\left\{B(t+1) \leq \Psi_{p}(B(t))\right\}$ is very likely. Since we think that $\Phi_{p}(k)$ defined in (2.1) is a 'sharp' conditional predictor of $B(t+1)$, we must have $\Psi_{p}(k)>\Phi_{p}(k)$. Also, to be of any use, $\Psi_{p}(k)$ must fall below $k$. Last, but not least, we must be able to solve a chosen recurrence. The function

$$
\Psi_{\boldsymbol{p}}(k)=\frac{1}{2}\left(k+\Phi_{\boldsymbol{p}}(k)\right)=\frac{1}{2}\left(k+n-F_{\boldsymbol{p}}(k)\right),
$$

with $F_{p}(k)$ as defined in (2.2), happens to meet all three requirements.

Define an event

$$
\Delta:=\left\{\text { for all } t: B(t) \geq k_{*} \text { implies that } B(t+1) \leq \Psi_{\mathbf{p}}(B(t))\right\} .
$$

In Subsection 2.5 we will show that $\mathrm{P}(\Delta) \rightarrow 1$ as $n \rightarrow \infty$. In this subsection, assuming that the event $\Delta$ holds, we solve the recurrence (2.17) and estimate sharply $\tau\left(k_{*}\right)$, the first moment $t$ when $B(t) \leq k_{*}$.

Lemma 2.1. On the event $\Delta$,

$$
\tau\left(k_{*}\right) \leq 5 c_{2}^{-1 / 2} \ln n=o\left(c_{2}^{-1}\right) .
$$

Proof. The proof is divided into two cases.

Case 1: $c_{2} \geq 2 n^{-1}$. First of all, by Proposition 2.2 we have

$$
\Psi_{\boldsymbol{p}}(k) \leq \Psi_{\boldsymbol{\theta}\left(c_{2}\right)}(k) \quad \text { for all } k \in[n],
$$

$\boldsymbol{\theta}\left(c_{2}\right)$ being the topheavy distribution $\left(\theta_{1}, \theta_{2}, \ldots, \theta_{2}\right)$ with parameter $c_{2}$. Therefore, on the event $\Delta$,

$$
B(t+1) \leq \Psi_{\boldsymbol{\theta}\left(c_{2}\right)}(B(t)) \text { for all } t \leq \tau\left(k_{*}\right) .
$$

Let us bound $\Psi_{\boldsymbol{\theta}\left(c_{2}\right)}(B(t))$ from above. Since $c_{2} \geq 2 n^{-1}$, we obtain, using (2.3),

$$
\theta_{1} \geq \frac{1}{2} c_{2}^{1 / 2} \Longrightarrow \quad \theta_{2} \leq \frac{1-(1 / 2) c_{2}^{1 / 2}}{n-1} .
$$

By (2.20) and

$$
\Psi_{\theta}(B(t))=\frac{1}{2}\left(B(t)+n-\exp \left(-\theta_{1} B(t)\right)-(n-1) \exp \left(-\theta_{2} B(t)\right)\right),
$$


we have (using the inequality $\mathrm{e}^{-x} \geq 1-x$ )

$$
\begin{aligned}
B(t+1) & \leq \frac{1}{2}\left(B(t)+n-0-(n-1)\left(1-\theta_{2} B(t)\right)\right) \\
& \leq \frac{1}{2}\left(B(t)+\left(1-\frac{c_{2}^{1 / 2}}{2}\right) B(t)+1\right) \\
& \leq\left(1-\frac{c_{2}^{1 / 2}}{4}\right) B(t)+\frac{1}{2},
\end{aligned}
$$

a linear recurrence inequality. (Implicit in this derivation is an intuition that, for the distribution $\boldsymbol{\theta}$ in question, a large enough proportion of collisions happen in box 1 and that we may disregard collisions in boxes $2, \ldots, n$ without inducing too large an error.) It follows, from (2.22) and $B(0)=n$, that

$$
B(t) \leq n\left(1-\frac{c_{2}^{1 / 2}}{4}\right)^{t}+2 c_{2}^{-1 / 2} \text { for } B(t) \geq k_{*} .
$$

To obtain a bound on $\tau\left(k_{*}\right)$, note that

$$
k_{*}<B\left(\tau\left(k_{*}\right)-1\right) \leq n\left(1-\frac{c_{2}^{1 / 2}}{4}\right)^{\tau\left(k_{*}\right)-1}+2 c_{2}^{-1 / 2},
$$

and let $\tau:=\tau\left(k_{*}\right)-1$. Now $c_{2}^{-1 / 2}=o\left(k_{*}\right)$ if $c_{2}=o\left(\ln ^{-2 \varepsilon} n\right)$, which is certainly implied by (1.4). So we can crudely use the bound $2 c_{2}^{-1 / 2} \leq \frac{1}{2} k_{*}$ in (2.24) to obtain

$$
\frac{1}{2} k_{*} \leq n\left(1-\frac{c_{2}^{1 / 2}}{4}\right)^{\tau} \leq n \exp \left(-\frac{c_{2}^{1 / 2} \tau}{4}\right)
$$

Taking logarithms and solving for $\tau$, we obtain

$$
\tau \leq 4\left(\ln n+\ln c_{2}+\varepsilon \ln \ln n+\ln 2\right) c_{2}^{-1 / 2} \leq 5 c_{2}^{-1 / 2} \ln n .
$$

Hence, $\tau\left(k_{*}\right)=o\left(c_{2}^{-1}\right)$, since $c_{2}=o\left(\ln ^{-2} n\right)$, which is the first condition in (1.4).

Case 2: $c_{2} \leq 2 n^{-1}$. This time $\boldsymbol{\theta}\left(c_{2}\right)$ is too close to being uniform, and inequality (2.21) is too crude. A bit of reflection shows that we should not expect $B(t)$ to decay exponentially here. We show instead that, for some absolute constant $A$,

$$
B(t) \leq \frac{A n}{t+1}, \quad t \leq \tau\left(k_{*}\right) .
$$

The proof is by induction. The case $t=0$ holds if $A \geq 1$. Suppose that (2.25) holds for some $t$. Observe that

$$
\Psi_{p}(k)=\sum_{j=1}^{n} \psi\left(p_{j} k\right), \quad \psi(x):=\frac{1}{2}\left(x+1-\mathrm{e}^{-x}\right),
$$

and that $\psi(x)$ is increasing and concave down. Then, by the inductive assumption,

$$
B(t+1) \leq \sum_{j=1}^{n} \psi\left(p_{j} B(t)\right) \leq \sum_{j=1}^{n} \psi\left(p_{j} \frac{A n}{t+1}\right) \leq n \psi\left(\frac{1}{n} \sum_{j=1}^{n} p_{j} \frac{A n}{t+1}\right)=n \psi\left(\frac{A}{t+1}\right) .
$$


So we need to find $A \geq 1$ such that

$$
\psi\left(\frac{A}{t+1}\right) \leq \frac{A}{t+2}
$$

or, defining $x:=A /(t+1)$,

$$
\psi(x) \leq x \frac{t+1}{t+2} \Longleftrightarrow 1-\mathrm{e}^{-x} \leq x\left(1-\frac{2}{t+2}\right) .
$$

We therefore define $x(t)$ implicitly by

$$
1-\mathrm{e}^{-x(t)}=x(t)\left(1-\frac{2}{t+2}\right)
$$

by considering the graphs of the functions of $x(t)$ on the left- and right-hand sides of (2.28), it is clear that $x(t)$ is well defined and decreasing (to 0 ) in $t$. Therefore, (2.27) is satisfied if and only if $x \geq x(t)$. It is not difficult to show that $x(t) \sim 4 /(t+2)$ as $t \rightarrow \infty$, and so $A_{*}:=\lim \sup _{t \rightarrow \infty}(t+1) x(t)$ is finite. Thus, to satisfy (2.26) and thereby to complete the inductive proof, we can pick $A=\max \left\{1, A_{*}\right\}$. Therefore, on the event $\Delta$, we have

$$
k_{*}<B\left(\tau\left(k_{*}\right)-1\right) \leq \frac{A n}{\tau\left(k_{*}\right)},
$$

which we can invert to obtain

$$
\tau\left(k_{*}\right) \leq \frac{A n}{k_{*}}=2 A \ln ^{\varepsilon} n,
$$

which is certainly $o\left(c_{2}^{-1 / 2} \ln n\right)$. Combining this with the case $c_{2} \geq 2 / n$, we have $\tau\left(k_{*}\right)=$ $O\left(c_{2}^{-1 / 2} \ln n\right)$ on the event $\Delta$. This completes the proof of Lemma 2.1.

\subsection{Exponential tail bounds}

We need to show that $\mathrm{P}(\Delta)$ converges to 1 and that it does so sufficiently fast. To this end, and also for Theorem 1.2, we establish two-sided exponential tail bounds for the distribution of $B(t+1)$ conditioned on $B(t)$. Like Chernoff bounds for sums of independent and identically distributed random variables, the bounds are based on a generating function approach for estimating the probabilities of large deviations.

Let $\pi_{k b}:=\mathrm{P}(B(t+1)=b \mid B(t)=k)$, and note that $\pi_{k b}=0$ for $k<b$. Introduce the tail probabilities

$$
\pi_{k b}^{-}=\mathrm{P}(B(t+1)<b \mid B(t)=k) \text { and } \pi_{k b}^{+}=\mathrm{P}(B(t+1)>b \mid B(t)=k) .
$$

Theorem 2.1. We have

$$
\pi_{k b}^{-} \leq 3 \sqrt{k} \exp \left(-\frac{\left(\Phi_{p}(k)-b\right)^{2}}{2 k}\right), \quad b \leq \Phi_{p}(k),
$$

and

$$
\pi_{k b}^{+} \leq 3 \sqrt{k} \exp \left(-\frac{\left(b-\Phi_{p}(k)\right)^{2}}{2 k}\right), \quad b \geq \Phi_{p}(k) .
$$

The heart of the proof is an expression for $\pi_{k b}$ by means of generating functions. As usual, the expression $\left[y^{m}\right] f(y)$ denotes the coefficient of $y^{m}$ in the power series expansion of $f(y)$. 
Lemma 2.2. We have

$$
\pi_{k b}=\frac{k !}{n^{k}}\left[x^{k} z^{b}\right] \prod_{j=1}^{n}\left(1+z\left(\exp \left(n p_{j} x\right)-1\right)\right), \quad 1 \leq b \leq k \leq n .
$$

Proof. We have

$$
\begin{aligned}
\pi_{k b} & =\mathrm{P}(B(t+1)=b \mid B(t)=k) \\
& =\sum_{U \subset[n],|U|=b} \mathrm{P}(k \text { balls go into exactly the boxes indexed by } U) \\
& =\sum_{U} \sum_{\substack{\varepsilon_{1}+\cdots+\varepsilon_{b}=k \\
\varepsilon_{j}>0 \text { for all } \\
j \in[b]}} \mathrm{P}\left(\text { each box } j \text { from } U \text { gets } \varepsilon_{j} \text { balls }\right) \\
& =\sum_{U} \sum_{\vec{\varepsilon}}\left(\begin{array}{c}
k \\
\varepsilon_{1} \varepsilon_{2} \cdots \varepsilon_{b}
\end{array}\right) \prod_{j \in U} p_{j}^{\varepsilon_{j}} \\
& =k ! \sum_{U} \sum_{\vec{\varepsilon}} \prod_{j \in U} \frac{p_{j}^{\varepsilon_{j}}}{\varepsilon_{j} !} .
\end{aligned}
$$

Now we build a bivariate generating function for the probabilities $\pi_{k b}$. Start with the $k$ index. Incorporating for future convenience an $n^{k}$ factor, we have

$$
\sum_{k} \pi_{k b} \frac{n^{k}}{k !} x^{k}=\sum_{U} \sum_{k} \sum_{\substack{\varepsilon_{1}+\cdots+\varepsilon_{b}=k \\ \varepsilon_{j}>0}} \prod_{j \in U} \frac{(n x)^{\varepsilon_{j}} p_{j}^{\varepsilon_{j}}}{\varepsilon_{j} !} .
$$

We merge the second and third sums, yielding for the right-hand side

$$
\sum_{U} \sum_{\varepsilon_{j}>0} \prod_{j \in U} \frac{(n x)^{\varepsilon_{j}} p_{j}^{\varepsilon_{j}}}{\varepsilon_{j} !}
$$

Reversing the order of summation and multiplication, we obtain

$$
\sum_{k} \pi_{k b} \frac{n^{k}}{k !} x^{k}=\sum_{U} \prod_{j \in U} \sum_{\varepsilon=1}^{\infty} \frac{\left(n x p_{j}\right)^{\varepsilon}}{\varepsilon !}=\sum_{U} \prod_{j \in U}\left(\exp \left(n p_{j} x\right)-1\right) .
$$

Multiplying by $z^{b}$ and summing for $b \geq 0$, we obtain

$$
\begin{aligned}
\sum_{b} \sum_{k} \pi_{k b} \frac{n^{k}}{k !} x^{k} z^{b} & =\sum_{b} z^{b} \sum_{|U|=b} \prod_{j \in U}\left(\exp \left(n p_{j} x\right)-1\right) \\
& =\sum_{U \subset[n]} z^{b} \prod_{j \in U}\left(\exp \left(n p_{j} x\right)-1\right) \\
& =\sum_{U \subset[n]} \prod_{j \in U} z\left(\exp \left(n p_{j} x\right)-1\right) \\
& =\prod_{j=1}^{n}\left(1+z\left(\exp \left(n p_{j} x\right)-1\right)\right) .
\end{aligned}
$$


Therefore, we have

$$
\pi_{k b} \frac{n^{k}}{k !}=\left[x^{k} z^{b}\right] \prod_{j=1}^{n}\left[1+z\left(\exp \left(n p_{j} x\right)-1\right)\right]
$$

and from here the lemma follows.

Proof of Theorem 2.1. By Lemma 2.2,

$$
g_{k}(z):=\mathrm{E}\left[z^{B(t+1)} \mid B(t)=k\right]=\sum_{b=1}^{k} \pi_{k b} z^{b}=\frac{k !}{n^{k}}\left[x^{k}\right] \prod_{j=1}^{n}\left(1+z\left(\exp \left(n p_{j} x\right)-1\right)\right) .
$$

Now, for $b \leq k$, we have

$$
\begin{aligned}
& z^{b} \pi_{k b}^{-}=\sum_{i=1}^{b} \pi_{k i} z^{b} \leq \sum_{i=1}^{b} \pi_{k i} z^{i} \leq g_{k}(z), \quad 0<z \leq 1, \\
& z^{b} \pi_{k b}^{+}=\sum_{i=b+1}^{k} \pi_{k i} z^{b} \leq \sum_{i=b+1}^{k} \pi_{k i} z^{i} \leq g_{k}(z), \quad z \geq 1 .
\end{aligned}
$$

This gives

$$
\begin{array}{ll}
\pi_{k b}^{-} \leq \frac{g_{k}(z)}{z^{b}}=\frac{k !}{z^{b} n^{k}}\left[x^{k}\right] \prod_{j=1}^{n}\left(1+z\left(\exp \left(n p_{j} x\right)-1\right)\right) & \text { for } 0<z \leq 1, \\
\pi_{k b}^{+} \leq \frac{g_{k}(z)}{z^{b}}=\frac{k !}{z^{b} n^{k}}\left[x^{k}\right] \prod_{j=1}^{n}\left(1+z\left(\exp \left(n p_{j} x\right)-1\right)\right) & \text { for } z \geq 1 .
\end{array}
$$

Since the coefficients of the products in (2.31)-(2.32) are nonnegative, we use the inequality $\left[x^{k}\right] f(x) \leq f(x) / x^{k}$ (for all $x>0$ ) to obtain

$$
\begin{array}{ll}
\pi_{k b}^{-} \leq \frac{k !}{z^{b}(n r)^{k}} \prod_{j=1}^{n}\left(1+z\left(\exp \left(n p_{j} r\right)-1\right)\right) & \text { for all } r>0 \text { and } 0<z \leq 1, \\
\pi_{k b}^{+} \leq \frac{k !}{z^{b}(n r)^{k}} \prod_{j=1}^{n}\left(1+z\left(\exp \left(n p_{j} r\right)-1\right)\right) & \text { for all } r>0 \text { and } z \geq 1 .
\end{array}
$$

Our task is to get the most out of bounds (2.33)-(2.34) by choosing values for $z$ and $r$ judiciously. We use Stirling's formula, $k ! \leq 3 \sqrt{k}(k / \mathrm{e})^{k}$, to transform the product-type formulae (2.33) and (2.34) into

$$
\begin{array}{ll}
\pi_{k b}^{-} \leq 3 \sqrt{k} \exp (H(z, r, b)), & z \leq 1 \\
\pi_{k b}^{+} \leq 3 \sqrt{k} \exp (H(z, r, b)), & z \geq 1
\end{array}
$$

where

$$
H(z, r, b):=k \ln \left(\frac{k}{r n \mathrm{e}}\right)-b \ln (z)+\sum_{j} \ln \left(1+z\left(\exp \left(n p_{j} r\right)-1\right)\right)
$$


For a given $b$, we want to use a stationary point of $H(z, r, b)$, i.e. a solution to

$$
\begin{aligned}
& H_{z}=-\frac{b}{z}+\sum_{j} \frac{\exp \left(n p_{j} r\right)-1}{1+z\left(\exp \left(n p_{j} r\right)-1\right)}=0, \\
& H_{r}=-\frac{k}{r}+z \sum_{j} \frac{n p_{j} \exp \left(n p_{j} r\right)}{1+z\left(\exp \left(n p_{j} r\right)-1\right)}=0 .
\end{aligned}
$$

This complicated system has a simple solution $\left(z_{*}, r_{*}\right)=(1, k / n)$ for

$$
b=b_{*}:=n-\sum_{j} \exp \left(-p_{j} k\right)\left(=\Phi_{p}(k)\right) .
$$

Moreover, from (2.37), it is immediate that $H\left(z_{*}, r_{*}, b_{*}\right)=0$. This is a first sign that the inequalities (2.35)-(2.36) may indeed lead to meaningful explicit bounds for $\pi_{k b}^{ \pm}$. Of course, we need to know that (2.38)-(2.39) has a solution $(z, r)$ for $b \neq b_{*}$ as well, such that $z<1$ for $b<b_{*}$ and $z>1$ for $b>b_{*}$.

Observe that

$$
\operatorname{det}\left(\begin{array}{ll}
H_{z z} & H_{z r} \\
H_{r z} & H_{r r}
\end{array}\right)=H_{z z} H_{r r}-H_{z r}^{2}>0
$$

for every solution $(z, r)$ of (2.38)-(2.39) (see Lemma A.1 in Appendix A for a proof of this). So, by the implicit vector function theorem, there exists an infinitely differentiable solution $(z(b), r(b))$ of (2.38)-(2.39) such that

$$
\left(z\left(b_{*}\right), r\left(b_{*}\right)\right)=\left(z_{*}, r_{*}\right) .
$$

Moreover, $z(b)$ is strictly increasing (see (A.13) in Appendix A), so that indeed $z(b)<1$ for $b<b_{*}$ and $z(b)>1$ for $b>b_{*}$. So, introducing

$$
h(b)=H(z(b), r(b), b),
$$

we have

$$
\begin{array}{ll}
\pi_{k b}^{-} \leq 3 \sqrt{k} \exp (h(b)), & b<b_{*}, \\
\pi_{k b}^{+} \leq 3 \sqrt{k} \exp (h(b)), & b>b_{*} ;
\end{array}
$$

here $h\left(b_{*}\right)=H\left(z_{*}, r_{*}, b_{*}\right)=0$.

To obtain efficient bounds from (2.41)-(2.42), let us approximate $h(b)$ by its Taylor polynomial about $b_{*}$. First, using (2.38)-(2.39),

$$
\begin{aligned}
h^{\prime}(b) & =\frac{\mathrm{d}}{\mathrm{d} b} H(z(b), r(b), b) \\
& =H_{z}(z(b), r(b), b) z^{\prime}(b)+H_{r}(z(b), r(b), b) r^{\prime}(b)+H_{b}(z(b), r(b), b) \\
& =H_{b}(z(b), r(b), b) \\
& =-\ln (z(b)) .
\end{aligned}
$$

It follows that $h(b)$ is unimodal (concave down, in fact), attaining its zero maximum at $b=b_{*}$. Consequently,

$$
h(b)=h\left(b_{*}\right)+h^{\prime}\left(b_{*}\right)\left(b-b_{*}\right)+\frac{h^{\prime \prime}(\tilde{b})}{2}\left(b-b_{*}\right)^{2}=\frac{h^{\prime \prime}(\tilde{b})}{2}\left(b-b_{*}\right)^{2},
$$


$\tilde{b}$ being between $b$ and $b_{*}$. It is shown in Appendix A (Lemma A.2) that

$$
h^{\prime \prime}(\tilde{b}) \leq-\frac{1}{k}
$$

This bound, (2.41), (2.42), and (2.44) imply (2.29) and (2.30), thereby concluding the proof of Theorem 2.1.

\subsection{Using the exponential tail bounds}

For the upper bound, we will need (2.30), which gives, for $b=\Psi_{p}(k)$ (recall the definition of $\Psi_{p}(k)$ in (2.18)),

$$
\begin{aligned}
\pi_{k, \Psi_{p}(k)}^{+} & \leq 3 \sqrt{k} \exp \left(-\frac{\left(\Psi_{p}(k)-\Phi_{p}(k)\right)^{2}}{2 k}\right) \\
& =3 \sqrt{k} \exp \left(-\frac{\left(k-\Phi_{p}(k)\right)^{2}}{8 k}\right) .
\end{aligned}
$$

Introducing

$$
H_{p}(k):=\frac{1}{k}\left(\Psi_{p}(k)-\Phi_{p}(k)\right)^{2}
$$

we rewrite (2.45) as

$$
\pi_{k, \Psi_{p}(k)}^{+} \leq 3 \sqrt{k} \exp \left(-\frac{1}{2} H_{p}(k)\right) .
$$

The next lemma states, roughly, that the larger $k$ is, the more likely it is that the next state $B(t+1)$ is close to the prediction based on information $B(t)=k$.

Lemma 2.3. For all $k$ and $\boldsymbol{p}, H_{p}(k)$ is increasing in $k$.

Proof. Define

$$
N_{p}(k):=\Psi_{p}(k)-\Phi_{p}(k)=\frac{1}{2}\left(k-n+\sum_{j=1}^{n} \exp \left(-p_{j} k\right)\right),
$$

so that $H_{\boldsymbol{p}}(k)=k^{-1} N_{\boldsymbol{p}}(k)^{2}$. Then

$$
H_{\boldsymbol{p}}^{\prime}(k)=\frac{N_{\boldsymbol{p}}(k)}{k^{2}}\left(2 k N_{\boldsymbol{p}}^{\prime}(k)-N_{\boldsymbol{p}}(k)\right),
$$

where

$$
2 k N_{\boldsymbol{p}}^{\prime}(k)-N_{\boldsymbol{p}}(k)=\frac{1}{2} \sum_{j=1}^{n}\left(p_{j} k+1-\left(2 p_{j} k+1\right) \exp \left(-p_{j} k\right)\right)>0
$$

because

$$
f(x):=x+1-(2 x+1) \mathrm{e}^{-x}>x+1-\frac{2 x+1}{x+1}=\frac{x^{2}}{x+1}>0 \text { for } x>0 .
$$

This completes the proof.

Lemma 2.4. For all $k \in\left\{k_{*}, \ldots, n\right\}$,

$$
H_{p}(k) \geq A_{*} \ln ^{1+\varepsilon} n,
$$

where $A_{*}$ is some absolute constant. Thus, the probability in (2.46) is superpolynomially small. 
Proof. Consider first the case in which $c_{2} \geq 2 n^{-1}$. From Proposition 2.3 and Lemma 2.3, it follows that, for all $k \geq k_{*}, H_{p}(k) \geq H_{p}\left(k_{*}\right) \geq H_{\boldsymbol{r}}\left(k_{*}\right)$; here

$$
\begin{aligned}
H_{\boldsymbol{r}}\left(k_{*}\right) & =\frac{\left(\Psi_{\boldsymbol{r}}\left(k_{*}\right)-\Phi_{\boldsymbol{r}}\left(k_{*}\right)\right)^{2}}{k_{*}} \\
& =\frac{1}{4 k_{*}}\left(\sum_{j=1}^{n}\left(\exp \left(-r_{j} k_{*}\right)-1+r_{j} k_{*}\right)\right)^{2} \\
& \geq \frac{1}{4 k_{*}}\left(v\left(\exp \left(-r_{1} k_{*}\right)-1+r_{1} k_{*}\right)\right)^{2} .
\end{aligned}
$$

To bound (2.47) from below, we need to have sharp bounds for $r_{1}$ and $\nu$. Recalling the definition of $\boldsymbol{r}$ in Lemma 2.3, and letting $\mu:=n-v-1$, we have

$$
v r_{1}+r_{2}+\mu r_{3}=1, \quad v r_{1}^{2}+r_{2}^{2}+\mu r_{3}^{2}=c_{2}, \quad v r_{1}^{3}+r_{2}^{3}+\mu r_{3}^{3}=c_{3} .
$$

Obviously, $r_{3} \leq n^{-1}$. Since we assume that $c_{2} \geq 2 n^{-1}$, we also have

$$
c_{3} \geq c_{2}^{2} \geq \frac{4}{n^{2}} \text {. }
$$

Hence,

$$
\mu r_{3}^{2} \leq \frac{\mu}{n^{2}} \leq \frac{1}{n} \leq \frac{c_{2}}{2}, \quad \mu r_{3}^{3} \leq \frac{\mu}{n^{3}} \leq \frac{1}{n^{2}} \leq \frac{c_{3}}{4} .
$$

Combining (2.48), (2.49), and $r_{2} \leq r_{1}$, we obtain

$$
\frac{c_{2}}{4} \leq v r_{1}^{2} \leq c_{2}, \quad \frac{3 c_{3}}{8} \leq v r_{1}^{3} \leq c_{3} .
$$

These double inequalities directly imply that

$$
\frac{3}{8} \frac{c_{3}}{c_{2}} \leq r_{1} \leq 4 \frac{c_{3}}{c_{2}}, \quad \frac{1}{64} \frac{c_{2}^{3}}{c_{3}^{2}} \leq v \leq \frac{64}{9} \frac{c_{2}^{3}}{c_{3}^{2}} .
$$

Armed with (2.51) we return to (2.47). Recalling that $k_{*}=c_{2}^{-1} \ln ^{-\varepsilon} n$, we need to consider separately the subsequences $\left\{n_{i}\right\}$ such that $r_{1} k_{*}=O(1)$ for $n \in\left\{n_{i}\right\}$, and the subsequences $\left\{n_{i}\right\}$ along which $r_{1} k_{*} \rightarrow \infty$. In the first case,

$$
\inf _{n_{i}} \frac{\exp \left(-r_{1} k_{*}\right)-1+r_{1} k_{*}}{r_{1}^{2} k_{*}^{2}} \geq \delta>0 .
$$

So, using (2.50), we obtain, from (2.47),

$$
H_{r}\left(k_{*}\right) \geq \frac{\delta^{2}}{4} v^{2} r_{1}^{4} k_{*}^{3} \geq \frac{\delta^{2}}{4}\left(v r_{1}^{2}\right)^{2} c_{2}^{-3} \ln ^{-3 \varepsilon} n \geq \frac{\delta^{2}}{64} c_{2}^{-1} \ln ^{-3 \varepsilon} n .
$$

In the second case, for large enough $n_{i}$,

$$
\exp \left(-r_{1} k_{*}\right)-1+r_{1} k_{*} \geq \frac{1}{2} r_{1} k_{*} .
$$

So, using (2.50) again and (2.51),

$$
H_{r}\left(k_{*}\right) \geq \frac{1}{16} v^{2} r_{1}^{2} k_{*}=\frac{1}{16} v\left(v r_{1}^{2}\right) k_{*} \geq \frac{1}{2^{12}} \frac{c_{2}^{3}}{c_{3}^{2}} \ln ^{-\varepsilon} n .
$$


Therefore, there exists a constant $A>0$ such that, for large enough $n$,

$$
H_{r}\left(k_{*}\right) \geq A \min \left\{c_{2}^{-1} \ln ^{-3 \varepsilon} n, \frac{c_{2}^{3}}{c_{3}^{2}} \ln ^{-\varepsilon} n\right\},
$$

for if there were not, there would be a subsequence $\left\{r_{1} k_{*}\left(n_{j}\right)\right\}_{j \geq 1}$ along which this did not hold. This subsequence would then have a further subsequence which tended to $\infty$, or remained bounded, which would contradict the bound for one of the two cases established above. Since

$$
c_{3} \leq c_{2}^{3 / 2} \ln ^{-(1 / 2+\varepsilon)} n, \quad c_{2} \leq C \ln ^{-2} n
$$

for some large enough constant $C$, the last inequality leads to

$$
H_{\boldsymbol{r}}\left(k_{*}\right) \geq A \ln ^{1+\varepsilon} n
$$

for large enough $n$, as long as $\varepsilon<\frac{1}{4}$, which of course we may assume without loss of generality.

Now consider the case in which $c_{2} \leq 2 n^{-1}$. This time, by Proposition 2.1 and Lemma 2.3, for all $k \geq k_{*}=c_{2}^{-1} \ln ^{-\varepsilon} n$,

$$
H_{\boldsymbol{p}}(k) \geq H_{\boldsymbol{p}}\left(k_{*}\right) \geq H_{\boldsymbol{u}}\left(k_{*}\right)
$$

where

$$
H_{\boldsymbol{u}}\left(k_{*}\right)=\frac{1}{4 k_{*}}\left(k_{*}-n+n \mathrm{e}^{-k_{*} / n}\right)^{2} \geq \frac{k_{*}^{3}}{36 n^{2}} \geq \frac{1}{288} n \ln ^{-3 \varepsilon} n .
$$

(For the first inequality, we used

$$
\left.\mathrm{e}^{-x}-1+x \geq \frac{x^{2}}{2}-\frac{x^{3}}{6} \geq \frac{x^{2}}{3} \quad \text { for } x \in(0,1) .\right)
$$

Thus, $H_{p}(k)=\omega\left(\ln ^{1+\varepsilon} n\right)$ for this case, and this concludes the proof.

\subsection{Validation of the deterministic approximation}

Now that we have established the (superpolynomially) small bound for $\pi_{k, \Psi_{p}(k)}^{+}$, we can finally show that the event $\Delta$ is extremely likely indeed.

Lemma 2.5. For some constant $A>0$,

$$
\mathrm{P}(\Delta) \geq 1-12 n \ln n \exp \left(-A \ln ^{1+\varepsilon} n\right) \geq 1-n^{-K} \quad \text { for all } K>0 \text { and } n \geq n(K) .
$$

Remark. Borrowing a term from Knuth et al. [10], the event $\Delta$ holds quite surely (q.s.).

Proof of Lemma 2.5. Introduce the events

$$
C_{t}=\left\{B(s+1) \leq \Psi_{p}(B(s)) \text { for all } s<t, B(t+1)>\Psi_{p}(B(t)), B(t) \geq k_{*}\right\} ;
$$

that is, $C_{t}$ is the event that the recursive inequality $B(s+1) \leq \Psi_{p}(B(s))$ is violated at a state $B(t) \geq k_{*}$, and $t$ is the first such moment. Clearly,

$$
\Delta^{\mathrm{c}}=\bigcup_{t \geq 0} C_{t}
$$


Let us show that

$$
C_{t}=\varnothing \quad \text { for all } t \geq t_{*}:=4 c_{2}^{-1 / 2} \ln n .
$$

Suppose, on the contrary, that $C_{t_{1}} \neq \varnothing$ for some $t_{1}>t_{*}$. Then, by the definition of $C_{t_{1}}$, we have

$$
B(s+1) \leq \Psi_{p}(B(s)) \quad \text { for all } s \leq t_{*},
$$

and certainly $B\left(t_{*}\right) \geq k_{*}$. However, using this recurrence inequality exactly as in the derivation of (2.23), we must have

$$
\begin{aligned}
B\left(t_{*}\right) & \leq n\left(1-\frac{c_{2}^{1 / 2}}{4}\right)^{t_{*}}+2 c_{2}^{-1 / 2} \\
& \leq n \exp \left(-\left(4 c_{2}^{-1 / 2}\right)(\ln n)\left(\frac{c_{2}^{1 / 2}}{4}\right)\right)+2 c_{2}^{-1 / 2} \\
& =1+2 c_{2}^{-1 / 2} \\
& <k_{*},
\end{aligned}
$$

since $c_{2}=o\left(\ln ^{-2 \varepsilon} n\right)$. This is a contradiction. Thus, $\Delta^{\mathrm{c}}$ is a union of at most $t_{*}$ events $C_{t}$. Now, by (2.45) and Lemma 2.3, we have

$$
\mathrm{P}\left(C_{t}\right) \leq 3 n^{1 / 2} \exp \left(-\frac{H_{p}\left(k_{*}\right)}{2}\right), \quad t \geq 0,
$$

since $B(t) \geq k_{*}$ on $C_{t}$. Therefore,

$$
\begin{aligned}
\mathrm{P}\left(\Delta^{\mathrm{c}}\right) & =\mathrm{P}\left(\bigcup_{t \leq t_{*}} C_{t}\right) \\
& \leq \sum_{t \leq t_{*}} \mathrm{P}\left(C_{t}\right) \\
& \leq 3 t_{*} n^{1 / 2} \exp \left(-\frac{H_{p}\left(k_{*}\right)}{2}\right) \\
& =12 c_{2}^{-1 / 2} n^{1 / 2} \ln n \exp \left(-\frac{H_{p}\left(k_{*}\right)}{2}\right) \\
& \leq 12 n \ln n \exp \left(-\frac{H_{p}\left(k_{*}\right)}{2}\right) \\
& \leq 12 n \ln n \exp \left(-0.5 A_{*} \ln ^{1+\varepsilon} n\right) \quad \text { by Lemma 2.4, }
\end{aligned}
$$

and from here the lemma follows.

This completes a program we put forth at the end of the introduction. Combining Lemma 2.1 and Lemma 2.5, we have proved the following.

Lemma 2.6. Let $k_{*}=c_{2}^{-1} \ln ^{-\varepsilon} n$ for some however small $\varepsilon \in\left(0, \frac{1}{4}\right)$, and let $\tau\left(k_{*}\right)$ denote the random moment when $B(t)$ falls to or below $k_{*}$ for the first time. Then, for some constant $a>0$,

$$
\mathrm{P}\left(\tau\left(k_{*}\right) \leq a c_{2}^{-1 / 2} \ln n\right) \geq 1-n^{-K} \text { for all } K>0 \text { and } n \geq n(K) .
$$

In short, q.s., $\tau\left(k_{*}\right)=o\left(c_{2}^{-1}\right)$. 


\subsection{Bounding the expectation of $\tau\left(k_{*}\right)$}

Even though $\tau\left(k_{*}\right)=o\left(c_{2}^{-1}\right)$ q.s., proving that $\mathrm{E}\left[\tau\left(k_{*}\right)\right]=o\left(c_{2}^{-1}\right)$ as well is not straightforward, since we do not have a polynomial (worst-case) bound for $\tau\left(k_{*}\right)$. As a first step, introducing the event indicators $I_{\Delta}$ and $I_{\Delta^{\mathrm{c}}}$, we split $\mathrm{E}\left[\tau\left(k_{*}\right)\right]$ using $1=I_{\Delta}+I_{\Delta^{\mathrm{c}}}$ and bound the second summand via the Cauchy-Schwarz inequality:

$$
\begin{aligned}
\mathrm{E}\left[\tau\left(k_{*}\right)\right] & =\mathrm{E}\left[\tau\left(k_{*}\right) I_{\Delta}\right]+\mathrm{E}\left[\tau\left(k_{*}\right) I_{\Delta^{\mathrm{c}}}\right] \\
& \leq 5(\ln n) c_{2}^{-1 / 2}+\sqrt{\mathrm{E}\left[\tau^{2}\left(k_{*}\right)\right] \mathrm{E}\left[I_{\Delta^{\mathrm{c}}}^{2}\right]} \\
& \leq 5(\ln n) c_{2}^{-1 / 2}+\sqrt{\mathrm{E}\left[\tau^{2}\left(k_{*}\right)\right]} \sqrt{\mathrm{P}\left(\Delta^{\mathrm{c}}\right)} .
\end{aligned}
$$

It remains to show that $\mathrm{E}\left[\tau^{2}\left(k_{*}\right)\right]$ is at most polynomially large. To do so, introduce $T_{k}$, the random time the process $\{B(t)\}$ spends at state $k$, i.e.

$$
T_{k}=|\{t \geq 0: B(t)=k\}| .
$$

Then

$$
\tau^{2}\left(k_{*}\right)=\left(\sum_{k=k_{*}+1}^{n} T_{k}\right)^{2} \leq\left(n-k_{*}\right) \sum_{k=k_{*}+1}^{n} T_{k}^{2},
$$

again by the Cauchy-Schwarz inequality. Therefore,

$$
\mathrm{E}\left[\tau^{2}\left(k_{*}\right)\right] \leq n \sum_{k=k_{*}+1}^{n} \mathrm{E}\left[T_{k}^{2}\right] \leq n \sum_{k=k_{*}+1}^{n} \mathrm{E}\left[T_{k}^{2} \mid T_{k}>0\right] .
$$

Recalling the notation $\pi_{k k}=\mathrm{P}(B(t+1)=k \mid B(t)=k)$, we observe that

$$
\mathrm{P}\left(T_{k}=j \mid T_{k}>0\right)=\pi_{k k}^{j-1}\left(1-\pi_{k k}\right), \quad j>0,
$$

i.e. conditioned on $\left\{T_{k}>0\right\}, T_{k}$ is geometrically distributed, with success probability $1-\pi_{k k}$. In particular,

$$
\mathrm{E}\left[T_{k} \mid T_{k}>0\right]=\frac{1}{1-\pi_{k k}}, \quad \operatorname{var}\left[T_{k} \mid T_{k}>0\right]=\frac{\pi_{k k}}{\left(1-\pi_{k k}\right)^{2}} .
$$

It is obvious intuitively, and can be easily proved, that $\pi_{k k}$ decreases with $k$ : the larger the number of balls - the larger the probability of collision. Consequently, both conditional moments of $T_{k}$ decrease with $k$. So

$$
\begin{aligned}
\mathrm{E}\left[\tau^{2}\left(k_{*}\right)\right] & \leq n \sum_{k=k_{*}+1}^{n}\left(\frac{\pi_{k k}}{\left(1-\pi_{k k}\right)^{2}}+\frac{1}{\left(1-\pi_{k k}\right)^{2}}\right) \\
& \leq n \sum_{k=k_{*}+1}^{n} \frac{2}{\left(1-\pi_{k k}\right)^{2}} \\
& \leq \frac{2 n^{2}}{\left(1-\pi_{k_{*} k_{*}}\right)^{2}}
\end{aligned}
$$


Therefore, it remains to show that only $1 /\left(1-\pi_{k_{*} k_{*}}\right)$ is at most polynomially large in $n$. Using the simplest lower bound for the probability of the union of events, via the inclusion-exclusion formula, we write

$$
\begin{aligned}
1-\pi_{k k} & =\mathrm{P}(\text { there is a collision during a } k \text {-allocation }) \\
& \geq \sum_{\{a, b\} \subset[k]} \mathrm{P}(\text { balls } a \text { and } b \text { collide })-\sum_{\{c, d\} \neq\{e, f\} \subset[k]} \mathrm{P}(c, d \text { collide and } e, f \text { collide }) \\
& \geq\left(\begin{array}{l}
k \\
2
\end{array}\right) c_{2}-\left(\left(\begin{array}{l}
k \\
3
\end{array}\right) c_{3}+\frac{1}{2}\left(\begin{array}{l}
k \\
2
\end{array}\right)\left(\begin{array}{c}
k-2 \\
2
\end{array}\right) c_{2}^{2}\right) \\
& =\left(\begin{array}{l}
k \\
2
\end{array}\right) c_{2}\left(1-a_{1} k \frac{c_{3}}{c_{2}}-a_{2} k^{2} c_{2}\right)
\end{aligned}
$$

where $a_{1}$ and $a_{2}$ are some absolute constants. The two rightmost terms of (2.55) are due to the fact that there are two ways in which two distinct pairs of balls can collide: either the two pairs overlap at one ball or they are disjoint. Now introduce $k_{1}:=c_{2}^{-1 / 2} \ln ^{-\varepsilon / 4} n$; clearly, $k_{1}<k_{*}$, and we also have

$$
\lim k_{1} \frac{c_{3}}{c_{2}}=0, \quad \lim k_{1}^{2} c_{2}=0 .
$$

So, by (2.56), uniformly for all $k \leq k_{1}$,

$$
\frac{1}{1-\pi_{k k}} \leq \frac{1}{\left(\begin{array}{c}
k \\
2
\end{array}\right) c_{2}}(1+o(1))
$$

Consequently,

$$
\frac{1}{1-\pi_{k_{*} k_{*}}} \leq \frac{1}{1-\pi_{k_{1} k_{1}}}=O\left(k_{1}^{-2} c_{2}^{-1}\right)=O\left(\ln ^{\varepsilon / 2} n\right)
$$

which is sufficient. Thus, by (2.52) and (2.54),

$$
\mathrm{E}\left[\tau\left(k_{*}\right)\right]=O\left(c_{2}^{-1 / 2} \ln n\right)=o\left(c_{2}^{-1}\right)
$$

\section{Bounding expected duration of a middle phase and a late phase}

To complete the proof of Theorem 1.1, it remains to bound the expected duration of the process after the number of balls has dropped below $k_{*}$.

We define a middle phase as $\left[\tau\left(k_{*}\right), \tau\left(k_{1}\right)\right)$, the time interval during which the number of balls is below $k_{*}$ and above $k_{1}$. Using the $T_{k}$ defined in (2.53), we have

$$
\mathrm{E}\left[\tau\left(k_{1}\right)-\tau\left(k_{*}\right)\right]=\sum_{k=k_{1}+1}^{k_{*}} \mathrm{E}\left[T_{k}\right] \leq \sum_{k=k_{1}+1}^{k_{*}} \frac{1}{1-\pi_{k k}} .
$$

Then, by decreasing monotonicity of $\left(1-\pi_{k k}\right)^{-1}$ and (2.58),

$$
\mathrm{E}\left[\tau\left(k_{1}\right)-\tau\left(k_{*}\right)\right] \leq \frac{k_{*}-k_{1}}{1-\pi_{k_{1} k_{1}}}=O\left(c_{2}^{-1} \ln ^{-\varepsilon+\varepsilon / 2} n\right)=O\left(c_{2}^{-1} \ln ^{-\varepsilon / 2} n\right)=o\left(c_{2}^{-1}\right) .
$$

(The last computation explains at long last why we needed the $\ln ^{-\varepsilon} n$ factor in the definition of $k_{*},(2.16)$.) 
Naturally, we define a late phase as $\left[\tau\left(k_{1}\right), \tau(1)\right]$. By (2.57),

$$
\mathrm{E}\left[\tau(1)-\tau\left(k_{1}\right)\right] \leq 1+\sum_{k=2}^{k_{1}} \frac{1+o(1)}{c_{2}\left(\begin{array}{l}
k \\
2
\end{array}\right)}=2 c_{2}^{-1}(1+o(1)),
$$

where we used

$$
\frac{1}{k(k-1)}=\frac{1}{k-1}-\frac{1}{k}, \quad k \geq 2 .
$$

That does it! Adding the estimates (2.59), (3.1), and (3.2), we obtain

$$
\mathrm{E}[\tau(1)] \leq 2 c_{2}^{-1}(1+o(1))
$$

\section{Lower bound}

We now provide the matching lower bound for Theorem 1.1. We will not need assumptions on $\boldsymbol{p}$ as strong as (1.4); rather, we will simply assume that $c_{2}(\boldsymbol{p}) \rightarrow 0$.

For any $m \leq n$, let $T(m)$ denote the coalescence time for the process starting with $m$ balls (we have so far been considering the case in which $T:=T(n)$.) We start by stating Theorem 2 of [1]: for any $\boldsymbol{p}$,

$$
\mathrm{E}[T(m)] \geq 2 c_{2}^{-1}\left(1-\frac{1}{m}-\frac{(m-1)(m-2)}{12} \frac{c_{3}}{c_{2}}\right) .
$$

To obtain a bound on $T(n)$, first note the following obvious-looking fact.

Proposition 4.1. Let $m_{1} \leq m_{2} \leq n$. Then, for any $\boldsymbol{p}, T\left(m_{2}\right)$ stochastically dominates $T\left(m_{1}\right)$.

Proof. This is a result of the following basic coupling argument. Start with $m_{2}$ balls, $m_{1}$ of which are marked, then perform the usual allocation process; the time $X$ at which all balls coalesce is distributed as $T\left(m_{2}\right)$, and at this time, certainly all marked balls have coalesced as well; call the time that these marked balls have first coalesced $Y$, so that $X \geq Y$ and $Y$ is distributed as $T\left(m_{1}\right)$. From here, the result follows.

This result shows that, for the bound $\mathrm{E}[T(n)] \geq 2 c_{2}^{-1}(1-o(1))$, it is sufficient to show that $\mathrm{E}[T(m)] \geq 2 c_{2}^{-1}(1-o(1))$ for some $m \leq n$. By the inequalities

$$
c_{2}^{2} \leq c_{3} \leq c_{2}^{3 / 2} \Longrightarrow c_{2} \leq \frac{c_{3}}{c_{2}} \leq c_{2}^{1 / 2},
$$

we have $c_{2} \rightarrow 0$ if and only if $c_{3} / c_{2} \rightarrow 0$. Let $m_{*}:=\left(c_{2} / c_{3}\right)^{1 / 3}$, say; then $m_{*} \rightarrow \infty$ since we assume that $c_{2} \rightarrow 0$, and so (4.1) becomes

$$
\mathrm{E}\left[T\left(m_{*}\right)\right] \geq 2 c_{2}^{-1}\left(1-\frac{1}{m_{*}}-O\left(m_{*}^{2} m_{*}^{-3}\right)\right)=2 c_{2}^{-1}(1-o(1)),
$$

and the result follows by Proposition 4.1. The proof of Theorem 1.1 is complete. 


\section{Proof of Theorem 1.2}

We start by restating Theorem 1.2 in a more detailed manner.

Theorem 5.1. Let $b_{0}=n$. Suppose that $c_{2}=\left(\ln ^{-2} n\right) \lambda(n)$, where $\lambda(n) \rightarrow \infty$ however slowly. Set $\boldsymbol{p}=\boldsymbol{\theta}\left(c_{2}\right)$, i.e. $\boldsymbol{p}$ is the topheavy distribution $\left(\theta_{1}, \theta_{2}, \ldots, \theta_{2}\right)$ with

$$
\theta_{1}^{2}+(n-1) \theta_{2}^{2}=c_{2} .
$$

Then, for the process evolving according to this p, w.h.p.,

$$
\tau(1) \geq c_{2}^{-1} \frac{\sqrt{\lambda(n)}}{20} ;
$$

so, in particular, $\mathrm{E}[\tau(1)]=\omega\left(c_{2}^{-1}\right)$.

Remarks. 1. Our choice of $\boldsymbol{p}$ should be expected. Indeed, the recurrence inequality (2.19) signals, intuitively, that the coalescent process for $\boldsymbol{p}=\boldsymbol{\theta}\left(c_{2}\right)$ is a good candidate for being the slowest among all $\boldsymbol{p}$ with $\sum_{j} p_{j}^{2}=c_{2}$.

2. In Theorem 5 of [1], Adler et al. proved that, for $\max _{j} p_{j}$ bounded away from 0 , and the remaining $p_{j}$ uniformly small, the expected coalescence time exceeds $c_{2}^{-1}$ by a factor of $\ln n$.

\subsection{Proof of Theorem 5.1}

For simplicity, we let $c:=c_{2}$. For $\boldsymbol{p}=\boldsymbol{\theta}(c)$, the Markov chain is almost as simple as that for the uniform $\boldsymbol{p}$. Indeed, given $B(t)$, the number of balls that land in box $2, \ldots, n$ (call it $\hat{B}(t))$ is binomially distributed with parameters $B(t)$ and success probability $1-\theta_{1}$, i.e. $\hat{B}(t)=\operatorname{Bin}\left(B(t), 1-\theta_{1}\right)$ in short. Conditioned on $\hat{B}(t)$, we have a uniform allocation of $\hat{B}(t)$ balls among $n-1$ boxes $2, \ldots, n$. Also, for sufficiently large $B(t)$, w.h.p., $\hat{B}(t) \sim\left(1-\theta_{1}\right) B(t)$. So, based on our experience with deterministic approximations earlier in the paper, we should expect that-after fusing balls that landed in the same box-these $\hat{B}(t)$ balls give birth to about

$$
(n-1)\left(1-\exp \left(-\frac{\hat{B}(t)}{n-1}\right)\right) \sim(n-1)\left(1-\exp \left(-\frac{\left(1-\theta_{1}\right) B(t)}{n-1}\right)\right)
$$

balls for the next generation. All the balls that landed in box 1 , if there were any, will coalesce into one ball. Ignoring this box for now, we expect then that the process $\{B(t)\}$, w.h.p., 'closely' obeys a recurrence inequality of the form

$$
B(t+1) \geq(n-1) \varphi\left(\frac{\left(1-\theta_{1}\right) B(t)}{n-1}\right), \quad \varphi(x):=1-\mathrm{e}^{-x} .
$$

Here is a precise claim.

Lemma 5.1. Let $\gamma:=1-2 c^{1 / 2}<1-\theta_{1}$, and introduce

$$
\Psi(k)=(n-1) \eta\left(\frac{\gamma k}{n-1}\right), \quad \eta(x):=1.5 \varphi(x)-0.5 x .
$$

Then, for sufficiently large $n$,

$$
\mathrm{P}(B(t+1)<\Psi(B(t)) \mid B(t)=k) \leq \mathrm{e}^{-k c / 3}+\exp \left(\frac{-k^{3}}{73 n^{2}}\right) .
$$

Remark. We have $\eta(0)=0, \eta(x) \leq \varphi(x)$, and $\eta(x)$ is increasing for $x \leq \ln 3$. 
Proof of Lemma 5.1. Note first that

$$
B(t+1) \geq \mathcal{R}(t+1),
$$

where $\mathcal{R}(t+1)$ is the number of boxes among $2, \ldots, n$ that host at least one of $\hat{B}(t)$ balls. Denoting $\mathrm{P}(\{\cdot\} \mid B(t)=k)$ by $\mathrm{P}_{k}(\{\cdot\})$, we then have

$$
\begin{aligned}
\mathrm{P}_{k}(B(t+1)<\Psi(k)) & \leq \mathrm{P}_{k}(\mathcal{R}(t+1)<\Psi(k)) \\
& \leq \mathrm{P}_{k}(\mathcal{R}(t+1)<\Psi(k), \hat{B}(t) \geq \gamma k)+\mathrm{P}_{k}(\hat{B}(t)<\gamma k) .
\end{aligned}
$$

Now, denoting the cumulative distribution function of $\mathcal{R}(t+1)$ conditioned on $\{\hat{B}(t)=j\}$ by $F_{j}$, we have, for $j_{1}<j_{2}$,

$$
F_{j_{2}}(x) \leq F_{j_{1}}(x) \text { for all } x \geq 0 .
$$

(Informally, the fewer balls we allocate among the boxes $2, \ldots, n$, the fewer nonempty boxes we end up with.) Therefore, letting $\lceil x\rceil=\min \{k \in \mathbb{N}: k \geq x\}$,

$$
\begin{aligned}
\mathrm{P}_{k}(\mathcal{R}(t+1)<\Psi(k), \hat{B}(t) \geq \gamma k) & =\sum_{j \geq \gamma k} \mathrm{P}(\mathcal{R}(t+1)<\Psi(k) \mid \hat{B}(t)=j) \mathrm{P}_{k}(\hat{B}(t)=j) \\
& \leq \mathrm{P}(\mathcal{R}(t+1)<\Psi(k) \mid \hat{B}(t)=\lceil\gamma k\rceil) \sum_{j \geq \gamma k} \mathrm{P}_{k}(\hat{B}(t)=j) \\
& \leq \mathrm{P}(\mathcal{R}(t+1)<\Psi(k) \mid \hat{B}(t)=\lceil\gamma k\rceil) .
\end{aligned}
$$

Consequently,

$$
\mathrm{P}_{k}(B(t+1)<\Psi(k)) \leq \mathcal{P}_{1}+\mathcal{P}_{2},
$$

where

$$
\begin{aligned}
& \mathcal{P}_{1}:=\mathrm{P}_{k}(\mathcal{R}(t+1)<\Psi(k) \mid \hat{B}(t)=\lceil\gamma k\rceil), \\
& \mathcal{P}_{2}:=\mathrm{P}_{k}(\hat{B}(t)<\gamma k) .
\end{aligned}
$$

Using the Chernoff bound for the tail of the binomial distribution (see, for instance, [11]), we have

$$
\begin{aligned}
\mathcal{P}_{2} & =\mathrm{P}\left(\operatorname{Bin}\left(k, 1-\theta_{1}\right)<\gamma k\right) \\
& \leq\left.\exp \left(-\frac{k p \delta^{2}}{2}\right)\right|_{p=1-\theta_{1}, \delta=1-\gamma\left(1-\theta_{1}\right)^{-1}} \\
& \leq \mathrm{e}^{-k c / 3}
\end{aligned}
$$

as $\theta_{1} \sim c_{2}^{1 / 2}$, from the definitions of $\theta_{1}$ and $\theta_{2}$ in (2.3).

We now turn to $\mathcal{P}_{1}$. Applying Theorem 2.1 to the boxes set $\{2, \ldots, n\}$ and the uniform distribution $\boldsymbol{u}$ on this set, we obtain

$$
\mathcal{P}_{1} \leq 3 \sqrt{\gamma k} \exp \left(-\frac{\left(\Phi_{\mathbf{u}}(\gamma k)-\Psi(k)\right)^{2}}{2 \gamma k}\right) .
$$

Here, using the definition of $\eta(x)$,

$$
\begin{aligned}
\Phi_{u}(\gamma k)-\Psi(k) & =(n-1) \varphi\left(\frac{\gamma k}{n-1}\right)-(n-1) \eta\left(\frac{\gamma k}{n-1}\right) \\
& =\frac{(n-1)}{2}\left(\frac{\gamma k}{n-1}-\varphi\left(\frac{\gamma k}{n-1}\right)\right) .
\end{aligned}
$$


So, as

$$
x-\varphi(x)=\mathrm{e}^{-x}-1+x \geq \frac{x^{2}}{2}-\frac{x^{3}}{6} \geq \frac{x^{2}}{3}, \quad x \in[0,1],
$$

we have

$$
\mathcal{P}_{1} \leq 4 \sqrt{\gamma k} \exp \left(-\frac{(\gamma k)^{4}}{72 \gamma k(n-1)^{2}}\right) \leq \exp \left(\frac{-k^{3}}{73 n^{2}}\right) .
$$

The estimates (5.2)-(5.3) imply (5.1), thereby completing the proof of the lemma.

To continue, let $\hat{k}:=n^{3 / 4}$. Introduce two events,

$$
\Gamma:=\{\text { for all } t, B(t) \geq \hat{k} \text { implies that } B(t+1) \geq \Psi(B(t))\}
$$

and

$$
\Pi:=\left\{\text { there exists } t>c^{-3 / 2}: B(t) \geq \hat{k}\right\} .
$$

On the event $\Gamma, B(t)$ does not decrease 'too quickly' as long as $B(t)$ is above $\hat{k}$. On the event $\Pi, \tau(\hat{k}) \geq c^{-3 / 2}$, i.e. $\tau(\hat{k})=\omega\left(c^{-1}\right)$. Then, by Lemma 5.1 ,

$$
\begin{aligned}
\mathrm{P}\left(\Gamma^{\mathrm{c}} \cap \Pi^{\mathrm{c}}\right) & \leq \mathrm{P}\left(\bigcup_{t=0}^{c^{-3 / 2}}\{B(t+1)<\Psi(B(t)), B(t) \geq \hat{k}\}\right) \\
& \leq\left(1+c^{-3 / 2}\right)\left(\mathrm{e}^{-\hat{k} c / 3}+\exp \left(-\frac{\hat{k}^{3}}{73 n^{2}}\right)\right) \\
& =\left(1+c^{-3 / 2}\right)\left(\exp \left(\frac{-n^{3 / 4} c}{3}\right)+\exp \left(\frac{-n^{1 / 4}}{73}\right)\right) \\
& \rightarrow 0
\end{aligned}
$$

i.e. $\mathrm{P}(\Gamma \cup \Pi) \rightarrow 1$.

If we show that $\tau(\hat{k})=\omega\left(c^{-1}\right)$ on the event $\Gamma$ as well, we will then be able to claim that, w.h.p., $\tau(\hat{k})=\omega\left(c^{-1}\right)$, and the proof of Theorem 5.1 will be complete.

To do so, we observe that, on the event $\Gamma$,

$$
x(t+1) \geq \eta(\gamma x(t)), \quad x(t):=\frac{B(t)}{n-1}, \quad x(0)=1-(n-1)^{-1},
$$

as long as

$$
x(t) \geq \frac{\hat{k}}{n-1} \sim n^{-1 / 4} .
$$

Lemma 5.2. Let $n \geq 3$. Under the recurrence in (5.4),

$$
x(t) \geq \frac{2}{3} \frac{\gamma^{t}}{t+1} .
$$

Proof. The argument runs in parallel to that for the lower bound of $B(t)$ in (2.25). The base case $t=0$ is just

$$
x(0)=1-n^{-1} \geq \frac{2}{3} .
$$

Suppose that (5.5) holds for some $t$. Since $\eta(x)$ is increasing for $x \leq \ln 3$, (5.4) implies that

$$
x(t+1) \geq \eta\left(\gamma \frac{2}{3} \frac{\gamma^{t}}{t+1}\right)=\eta\left(\frac{2}{3} \frac{\gamma^{t+1}}{t+1}\right) .
$$


So, to complete the inductive step, we need to show that

$$
\eta(y) \geq y \frac{t+1}{t+2}, \quad y:=\frac{2}{3} \frac{\gamma^{t+1}}{t+1} .
$$

Define $z$ as a root of

$$
\eta(z)=z \frac{t+1}{t+2} \quad \text { or } \quad 1-\mathrm{e}^{-z}=z \frac{t+4 / 3}{t+2} .
$$

Since $1-\mathrm{e}^{-1}<\frac{2}{3},(5.7)$ has a (unique) root $z=z(t)$ for $t \geq 0$. Using $1-\mathrm{e}^{-z} \geq z-z^{2} / 2$, we obtain

$$
z(t) \geq \frac{4 / 3}{t+2}
$$

Inequality (5.6) holds if $y \leq z(t)$, which is certainly so because

$$
y(t) \leq \frac{2 / 3}{t+1} \leq \frac{4 / 3}{t+2} \text { for all } t \geq 0,
$$

and from here the lemma follows.

Thus, on the event $\Gamma$,

$$
B(t) \geq \hat{k} \quad \Longrightarrow \quad B(t+1) \geq(n-1) \frac{2}{3} \frac{\gamma^{t+1}}{t+2} .
$$

Lemma 5.3. On the event $\Gamma$,

$$
\tau(\hat{k}) \geq \frac{\ln ^{2} n}{20 \sqrt{\lambda(n)}}=c^{-1} \frac{\sqrt{\lambda(n)}}{20} .
$$

Proof. Let the event $\Gamma$ hold. By (5.8), and the definition of $\tau(\cdot), \hat{\tau}:=\tau(\hat{k})$ satisfies

$$
\hat{k} \geq B(\hat{\tau}) \geq(n-1) \frac{2}{3} \frac{\gamma^{\hat{\tau}}}{\hat{\tau}+1} .
$$

Recalling that $\hat{k}=n^{3 / 4}, \gamma=1-2 c^{1 / 2}$, and taking logarithms, we obtain

$$
3 c^{1 / 2} \hat{\tau}+\ln (\hat{\tau}+1) \geq \frac{1}{5} \ln n,
$$

or

$$
3 \frac{\sqrt{\lambda(n)}}{\ln n} \hat{\tau}+\ln (\hat{\tau}+1) \geq \frac{1}{5} \ln n .
$$

It then follows that, for large enough $n$,

$$
\hat{\tau} \geq \frac{1}{4 \cdot 5} \frac{\ln ^{2} n}{\sqrt{\lambda(n)}}=c^{-1} \frac{\sqrt{\lambda(n)}}{20},
$$

as if it did not then $\ln (\hat{\tau}+1)=o(\ln n)$ and

$$
3 \frac{\sqrt{\lambda(n)}}{\ln n} \hat{\tau} \leq \frac{3}{4 \cdot 5} \ln n<\ln n,
$$

which together contradict (5.9) for large enough $n$. This completes the proof. 
In summary, on the event $\Gamma \cup \Pi$,

$$
\tau(\hat{k}) \geq \min \left\{c^{-3 / 2}, c^{-1} \frac{\sqrt{\lambda(n)}}{20}\right\}=c^{-1} \frac{\sqrt{\lambda(n)}}{20} .
$$

Recalling that $\mathrm{P}(\Gamma \cup \Pi) \rightarrow 1$, we conclude that

$$
c \mathrm{E}[\tau(1)] \geq c \mathrm{E}[\tau(\hat{k})] \geq \mathrm{E}\left[\tau(\hat{k}) I_{\Gamma \cup \Pi}\right] \geq \frac{\sqrt{\lambda(n)}}{20} \mathrm{P}(\Gamma \cup \Pi) \rightarrow \infty .
$$

This concludes the proof of Theorem 5.1.

\section{Concluding remarks and future work}

A generalization of this problem is to allow the probability of a ball going to a certain box to depend on its origin and not just on its destination; that is, if we have a ball in box $i$, it has probability $p_{i j}$ of landing in box $j$ for any $j \in[n]$, and these probabilities are not necessarily the same for all $i$. This is more difficult, as $\{B(t)\}_{t \geq 0}$ is no longer a Markov chain: we have to keep track of the locations of the balls at any time and not simply their number.

Coupling from the past algorithms involve running simultaneous coalescing flows on a Markov chain $\boldsymbol{P}:=\left(p_{i j}\right)_{1 \leq i, j \leq n}$ with stationary distribution $\boldsymbol{\pi}$, and return samples distributed exactly according to $\pi$, at the time when all the flows coalesce. It would be very interesting to extend the techniques in this paper to a more general case (when the rows of $\boldsymbol{P}$ are not necessarily all equal to some vector $\boldsymbol{p}$ ) in order to obtain an upper bound for the expected running time of such algorithms.

Here is an approach that appears promising. We start with a fixed allotment of one ball in each of the $n$ boxes and, for $t_{*}$ times, we run $n$ independent allocations for each ball, where we do not fuse balls that land in the same box; call this a mixing phase. This $t_{*}$ is to be taken large enough so that the location of each ball at time $t_{*}$ is 'almost' $\pi$-distributed. Then, at time $t_{*}+1$, we allocate the balls into the boxes but fuse any that collide (a fusing allocation). Continue alternating between mixing phases and fusing allocations until total coalescence has occurred; the coalescence time for this process should dominate the time for the usual process where we fuse colliding balls at every time. Moreover, if the locations of the balls at each fusing allocation are sufficiently independent and close to $\pi$-distributed, then we may be able to bring the results in this paper to bear on this more general case.

\section{Appendix A. Missing parts of the proof of Theorem 2.1}

Lemma A.1. Let $\chi=H_{r r} H_{z z}-H_{r z}^{2}$. Then $\chi>0$ for all $(z, r, b)$ on the curve $C$ defined by (2.38) and (2.39).

Proof. By the definition of $H$, we have

$$
\begin{gathered}
H_{z z}=\frac{b}{z^{2}}-\sum_{j} \frac{\left(\exp \left(n p_{j} r\right)-1\right)^{2}}{\left(1+z\left(\exp \left(n p_{j} r\right)-1\right)\right)^{2}}, \\
H_{r r}=\frac{k}{r^{2}}+z(1-z) n^{2} \sum_{j} \frac{p_{j}^{2} \exp \left(n p_{j} r\right)}{\left(1+z\left(\exp \left(n p_{j} r\right)-1\right)\right)^{2}},
\end{gathered}
$$


and

$$
H_{r z}=n \sum_{j} \frac{p_{j} \exp \left(n p_{j} r\right)}{\left(1+z\left(\exp \left(n p_{j} r\right)-1\right)\right)^{2}} .
$$

We can recast (2.38) and (2.39) as

$$
b=\sum_{j} \frac{z\left(\exp \left(n p_{j} r\right)-1\right)}{1+z\left(\exp \left(n p_{j} r\right)-1\right)}, \quad k=n r z \sum_{j} \frac{p_{j} \exp \left(n p_{j} r\right)}{1+z\left(\exp \left(n p_{j} r\right)-1\right)} .
$$

Using these in (A.1)-(A.3), we obtain, on $C$,

$$
\begin{gathered}
H_{z z}=\frac{1}{z^{2}} \sum_{j} \frac{z\left(\exp \left(n p_{j} r\right)-1\right)}{1+z\left(\exp \left(n p_{j} r\right)-1\right)}-\sum_{j} \frac{\left(\exp \left(n p_{j} r\right)-1\right)^{2}}{\left(1+z\left(\exp \left(n p_{j} r\right)-1\right)\right)^{2}}, \\
H_{r r}=\frac{n z}{r} \sum_{j} \frac{p_{j} \exp \left(n p_{j} r\right)}{1+z\left(\exp \left(n p_{j} r\right)-1\right)}+z(1-z) n^{2} \sum_{j} \frac{p_{j}^{2} \exp \left(n p_{j} r\right)}{\left(1+z\left(\exp \left(n p_{j} r\right)-1\right)\right)^{2}},
\end{gathered}
$$

and now (A.5) simplifies to

$$
H_{z z}=\frac{1}{z} \sum_{j} \frac{\left(\exp \left(n p_{j} r\right)-1\right)}{\left(1+z\left(\exp \left(n p_{j} r\right)-1\right)\right)^{2}}
$$

As for $H_{r r}$, the best we can do is to rewrite the equation in the form

$$
H_{r r}=\sum_{j} \frac{n z p_{j} \exp \left(n p_{j} r\right)\left(1+z\left(\exp \left(n p_{j} r\right)-1\right)\right)+r z(1-z) n^{2} p_{j}^{2} \exp \left(n p_{j} r\right)}{r\left(1+z\left(\exp \left(n p_{j} r\right)-1\right)\right)^{2}} .
$$

Using the inequality $\mathrm{e}^{x}-1 \geq x$ gives

$$
H_{r r} \geq \sum_{j} \frac{n z p_{j} \exp \left(n p_{j} r\right)\left(1+z n p_{j} r\right)+r z(1-z) n^{2} p_{j}^{2} \exp \left(n p_{j} r\right)}{r\left(1+z\left(\exp \left(n p_{j} r\right)-1\right)\right)^{2}}
$$

which then leads to some very convenient cancelling (in particular, of the $z^{2}$ term) to obtain

$$
H_{r r} \geq \sum_{j} \frac{n z p_{j} \exp \left(n p_{j} r\right)\left(1+r n p_{j}\right)}{r\left(1+z\left(\exp \left(n p_{j} r\right)-1\right)\right)^{2}}
$$

Multiplying (A.6) and (A.7) together, we obtain

$$
H_{z z} H_{r r} \geq \frac{n}{r}\left(\sum_{j} \frac{\left(\exp \left(n p_{j} r\right)-1\right)}{\left(1+z\left(\exp \left(n p_{j} r\right)-1\right)\right)^{2}}\right)\left(\sum_{j} \frac{p_{j} \exp \left(n p_{j} r\right)\left(1+r n p_{j}\right)}{\left(1+z\left(\exp \left(n p_{j} r\right)-1\right)\right)^{2}}\right),
$$

and using the Cauchy-Schwarz inequality, we obtain

$$
H_{z z} H_{r r} \geq \frac{n}{r}\left(\sum_{j} \frac{\left[\left(\exp \left(n p_{j} r\right)-1\right) p_{j}\left(1+n p_{j} r\right) \exp \left(n p_{j} r\right)\right]^{1 / 2}}{\left(1+z\left(\exp \left(n p_{j} r\right)-1\right)\right)^{2}}\right)^{2} .
$$


We need to show that this is strictly greater than $H_{r z}^{2}$, which can be expressed, using (A.3), as

$$
H_{r z}^{2}=n^{2}\left(\sum_{j} \frac{p_{j} \exp \left(n p_{j} r\right)}{\left(1+z\left(\exp \left(n p_{j} r\right)-1\right)\right)^{2}}\right)^{2} .
$$

Taking square roots of the expressions in (A.8) and (A.9), the condition $\chi>0$ is equivalent to

$$
\sum_{j} \frac{\left[\left(\exp \left(n p_{j} r\right)-1\right) p_{j}\left(1+n p_{j} r\right) \exp \left(n p_{j} r\right)\right]^{1 / 2}-\sqrt{r n} p_{j} \exp \left(n p_{j} r\right)}{\left(1+z\left(\exp \left(n p_{j} r\right)-1\right)\right)^{2}}>0 .
$$

For (A.10) to hold, it suffices that each summand is nonnegative (and at least one strictly positive). Multiplying the numerators by $\sqrt{r n}$, we need to show that, for all $j, 1 \leq j \leq n$,

$$
\left[\left(\exp \left(n p_{j} r\right)-1\right) n p_{j} r\left(1+n p_{j} r\right) \exp \left(n p_{j} r\right)\right]^{1 / 2}-r n p_{j} \exp \left(n p_{j} r\right) \geq 0 .
$$

This is equivalent to showing that, for all $j$,

$$
f\left(n p_{j} r\right) \geq 0
$$

where we define $f(x)=\left(\mathrm{e}^{x}-1\right) x(1+x) \mathrm{e}^{x}-x^{2} \mathrm{e}^{2 x}$. Now

$$
\begin{aligned}
f(x) & =x \mathrm{e}^{2 x}+x^{2} \mathrm{e}^{2 x}-x \mathrm{e}^{x}-x^{2} \mathrm{e}^{x}-x^{2} \mathrm{e}^{2 x} \\
& =x \mathrm{e}^{2 x}-x \mathrm{e}^{x}-x^{2} \mathrm{e}^{x}=x \mathrm{e}^{x}\left(\mathrm{e}^{x}-1-x\right) \\
& >0 \text { for } x>0 .
\end{aligned}
$$

Therefore, the inequalities in (A.11) hold (and at least one of them is strict), and so the lemma follows.

Lemma A.2. With $h(b)$ as defined in (2.40), we have, uniformly for $b \leq k$,

$$
h^{\prime \prime}(b) \leq-\frac{1}{k}
$$

Proof. First note that

$$
\begin{aligned}
h^{\prime \prime}(b) & =\frac{\mathrm{d}}{\mathrm{d} b}\left(h^{\prime}(b)\right) \\
& =\frac{\mathrm{d}}{\mathrm{d} b}(-\ln (z(b))) \quad \text { by }(2.43) \\
& =-\frac{z^{\prime}(b)}{z(b)} .
\end{aligned}
$$

To find $z^{\prime}(b)$, differentiate $H_{z}(z(b), r(b), b)=0$ and $H_{r}(z(b), r(b), b)=0$ with respect to $b$; we can solve for $z^{\prime}(b)$ in this system (using $H_{r b}=0$ and $H_{z b}=-1 / z$ ) to obtain

$$
z^{\prime}(b)=\frac{H_{r r}}{z(b)\left(H_{z z} H_{r r}-H_{r z}^{2}\right)}=\frac{H_{r r}}{z(b) \chi}
$$


which is strictly positive by (A.7) and Lemma A.1. Now note that, using the expression for $H_{z z}$ in (A.6), and (A.4) to express $b$,

$$
\begin{aligned}
\frac{H_{z z}}{b} & =\frac{1}{z(b)^{2} b} \sum_{j} \frac{z(b)\left(\exp \left(n p_{j} r(b)\right)-1\right)}{\left[1+z(b)\left(\exp \left(n p_{j} r(b)\right)-1\right)\right]^{2}} \\
& \leq \frac{1}{z(b)^{2} b} \sum_{j} \frac{z(b)\left(\exp \left(n p_{j} r(b)\right)-1\right)}{1+z(b)\left(\exp \left(n p_{j} r(b)\right)-1\right)} \\
& =\frac{1}{z(b)^{2}} .
\end{aligned}
$$

Therefore, using (A.12), (A.13), and (A.14),

$$
h^{\prime \prime}(b)=-\frac{z^{\prime}(b)}{z(b)}=-\frac{H_{r r}}{z(b)^{2} \chi} \leq \frac{-H_{r r} H_{z z}}{b \chi} \leq-\frac{1}{b},
$$

where the last inequality holds because $0<\chi<H_{r r} H_{z z}$. Using the fact that $b \leq k, \Phi_{p}(k) \leq k$, and $\tilde{b}$ is between $b$ and $\Phi_{p}(k)$, we obtain

$$
h^{\prime \prime}(\tilde{b}) \leq-\frac{1}{k} .
$$

\section{Acknowledgements}

We are very grateful to the participants of a student workshop on combinatorial probability at The Ohio State University for many productive discussions of this project. We would also like to thank an anonymous reviewer for many insightful corrections and suggestions that greatly helped us improve the presentation of the paper.

The research of the first author was supported in part by an NSF VIGRE fellowship. The research of the second author was supported in part by an NSF grant.

\section{References}

[1] Adler, I., Ahn, H.-S., Karp, R. M. And Ross, S. M. (2003). Coalescing times for iid random variables with applications to population biology. Random Structures Algorithms 23, 155-166.

[2] Dalal, A. and Schmutz, E. (2002). Compositions of random functions on a finite set. Electron J. Combinatorics 9, 7 pp.

[3] Donnelly, P. and Tavaré, S. (1995). Coalescents and genealogical structure under neutrality. Ann. Rev. Genet. 29, 401-421.

[4] FILL, J. (2002). On compositions of random functions on a finite set. Unpublished manuscript. Available at http://www.mts.jhu.edu/ fill/papers/compositions.ps.

[5] Frieze, A. M. and Grimmett, G. R. (1985). The shortest-path problem for graphs with random arc-lengths. Discrete Appl. Math. 10, 57-77.

[6] Goh, W., Hitczenko, P. and Schmutz, E. (2006). Iterating random functions on a finite set. Unpublished manuscript. Available at http://front.math.ucdavis.edu/0207.5276.

[7] Kingman, J. F. C. (1982). Exchangeability and the evolution of large populations. In Exchangeability in Probability and Statistics, eds G. Koch and F. Spizzichino, North-Holland, Amsterdam, pp. 97-112.

[8] Kingman, J. F. C. (1982). On the genealogy of large populations. In Essays in Statistical Science (J. Appl. Prob. Spec. Vol. 19A), eds J. Gani and E. J. Hannan, Applied Probability Trust, pp. 27-43.

[9] Kingman, J. F. C. (1982). The coalescent. Stoch. Process Appl. 13, 235-248.

[10] Knuth, D. E., Motwani, R. And Pittel, B. (1990). Stable husbands. Random Structures Algorithms 1, 1-14.

[11] Mitzenmacher, M. and Upfal, E. (2005). Probability and Computing. Cambridge University Press.

[12] MöHLe, M. (1998). Robustness results for the coalescent. J. Appl. Prob. 35, 438-447. 
[13] Möhle, M. (2004). The time back to the most recent common ancestor in exchangeable population models. Adv. Appl. Prob. 36, 78-97.

[14] Möhle, M. And Sagitov, S. (2001). A classification of coalescent processes for haploid exchangeable population models. Ann. Prob. 29, 1547-1562.

[15] Pitman, J. (1999). Coalescents with multiple collisions. Ann. Prob. 27, 1870-1902.

[16] Pittel, B. (1987). On spreading a rumor. SiAM J. Appl. Math. 47, 213-223.

[17] Propp, J. G. and Wilson, D. B. (1996). Exact sampling with coupled Markov chains and applications to statistical mechanics. Random Structures Algorithms 9, 223-252.

[18] Propp, J. G. AND Wilson, D. B. (1998). How to get a perfectly random sample from a generic Markov chain and generate a random spanning tree of a directed graph. J. Algorithms 27, 170-217.

[19] Sagitov, S. (1999). The general coalescent with asynchronous mergers of ancestral lines. J. Appl. Prob. 36, 1116-1125.

[20] Schweinsberg, J. (2000). Coalescents with simultaneous multiple collisions. Electron. J. Prob. 5, 50 pp. 